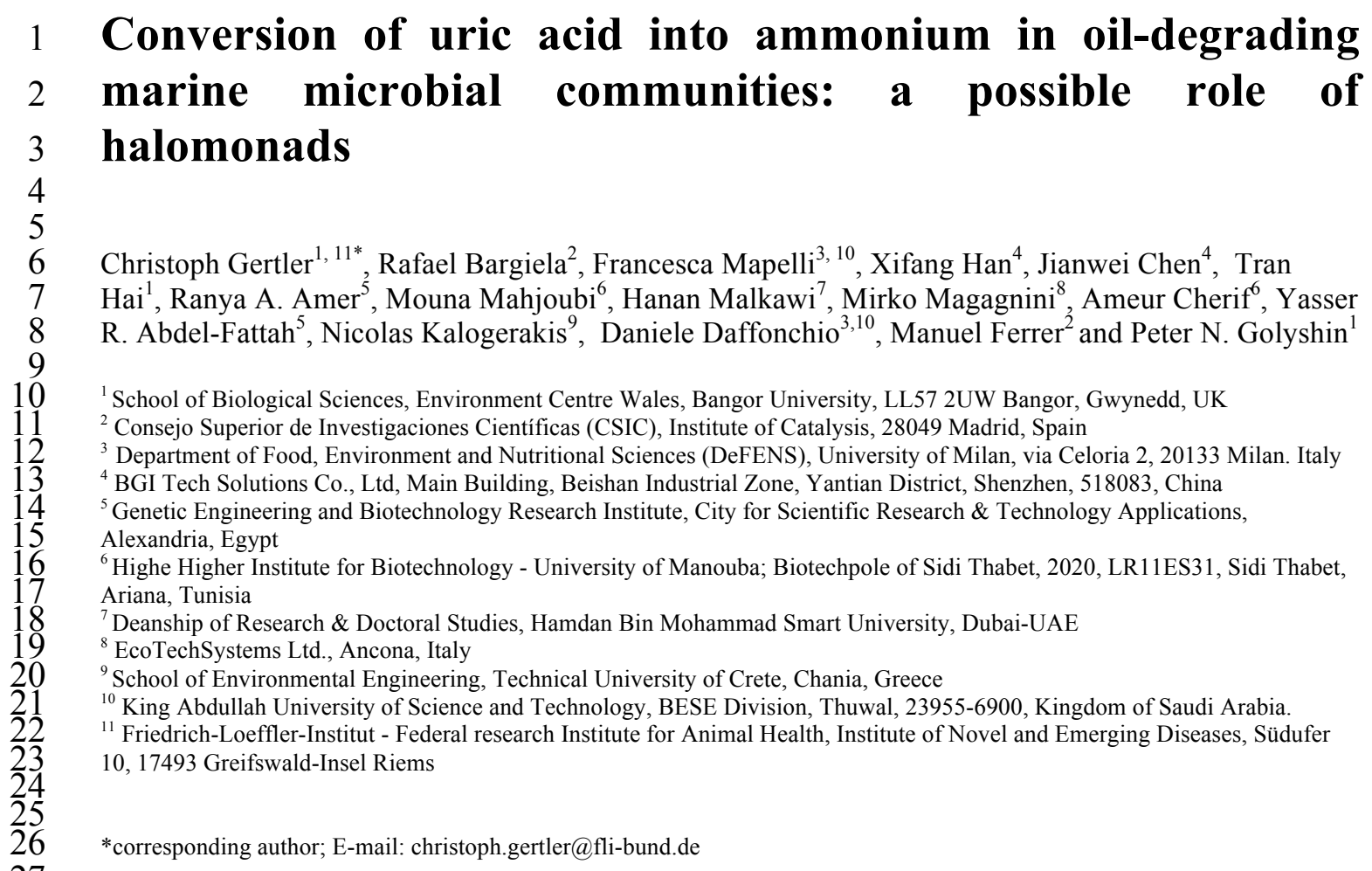

Abstract

Uric acid is a promising hydrophobic nitrogen source for biostimulation of microbial activities in oil-impacted marine environments. This study investigated metabolic processes and microbial community changes in a series of microcosms using sediment from the Mediterranean and the Red Sea amended with ammonium and uric acid. Respiration, emulsification, ammonium and protein concentration measurements suggested a rapid production of ammonium from uric acid accompanied by the development of microbial communities containing hydrocarbonoclastic bacteria after 3 weeks of incubation. About $80 \%$ of uric acid was converted to ammonium within the first few days of the experiment. Microbial population dynamics were investigated by Ribosomal Intergenic Spacer Analysis and Illumina sequencing as well as by culture-based techniques. Resulting data indicated that strains related to Halomonas spp. converted uric acid into ammonium, which stimulated growth of microbial consortia dominated by Alcanivorax spp. and Pseudomonas spp. Several strains of Halomonas spp. were isolated on uric acid as the sole carbon source showed location specificity. These results point towards a possible role of halomonads in the conversion of uric acid to ammonium utilized by hydrocarbonoclastic bacteria. 
3 Microbial hydrocarbon degradation in the sea occurs on a global scale in a large variety of latitudinal zones and at all ocean depths. More than five decades of research have shown that marine oil degrading microbes are highly specialised with regard to their substrate spectrum and climate zone (Head et al., 2006; Yakimov et al., 2007). Cold-adapted microbes such as Oleispira spp. prevail in cold polar zones and in the deep sea whereas oil degrading consortia in temperate zones are dominated by Alcanivorax species (Kube et al., 2013) in the aftermath of major oil spills. Many members of Oceanospirillales and Alteromonadales (e.g. Thalassolituus spp. and Marinobacter spp.) become predominant in the early stages of oildegrading microbial consortia due to their highly adapted metabolism (Kasai et al., 2002; Yakimov et al., 2004). Late stages of marine hydrocarbonoclastic consortia on the other hand are characterized by bacteria such as Cycloclasticus spp. specialized in aromatic and polyaromatic hydrocarbons that are degraded in a slower process (Head et al., 2006).

To perform hydrocarbon degradation, microbes depend upon nitrogen and, to a lesser extent, phosphorus sources (Atlas and Bartha, 1972). Both elements are scarce in seawater in most parts of the oceans and over most of the warmer months due to competition for nutrients by phototrophs that use sunlight and $\mathrm{CO}_{2}$ as energy and carbon sources. In fact, dissolved organic nitrogen, ammonium and nitrate exist in mere trace amounts after phyoplankton spring blooms from March to June and are recycled in winter months when low temperatures prohibit fast growth of heterotrophic microbes (Garcia et al., 2010). The artificial addition of nitrogen and phosphorus sources into an oil impacted environment on the other hand leads to a quick growth of hydrocarbonoclastic microbes and substantial microbial hydrocarbon degradation. An example of the successful implementation of this approach was the $M / V$ Exxon Valdez mitigation trial (Lindstrom et al., 1991). Studies of marine oil-degrading microbial communities growing in dispersed oil did not show a significant increase of oil degrading microbes beyond $10^{5}$ cells $/ \mathrm{mL}$ and no significant biogenic hydrocarbon degradation, either (Hazen et al., 2010).

29 A variety of different nitrogen sources can be used to support growth of hydrocarbonoclastic 30 bacteria (HCB). Oil spill remediation trials commonly use nitrogen in forms of ammonium, 31 nitrate, urea, uric acid and amino acids (Fava et al., 2004). Each nitrogen source has its advantages and disadvantages (Nikolopoulou and Kalogerakis, 2008): ammonium and nitrate are water-soluble and universally accepted by microbes, but they are toxic at high concentrations as well as prone to leaching and losses by denitrification or precipitation in seawater (Wrenn et al., 2001). Urea is water-soluble and widely accepted by microbes but easily leached. Uric acid is less widely accepted by microbes. Its oleophilic nature allows it to 
1 complex nitrogen and phosphorus source that has recently been used in aromatic hydrocarbon 2 degradation experiments (Fava, et al., 2004). While it is readily used and can be supplied in 3 large quantities, the mechanism of lecithin metabolism in PAH-degrading microbial consortia remains unknown. Furthermore, large amounts of lecithin must be applied due to the high $\mathrm{C}: \mathrm{N}$ ratio of the lecithin molecule. Certain industrial processes can overcome the disadvantages of some nitrogen sources e.g., sulphur coating of urea (Lee et al., 1993) or using vegetable oil coated ammonium/nitrate in slow release fertilizers (Gertler et al., 2009). This reduces the cost efficiency of such nitrogen sources in bioremediation trials, though. Uric acid could be used without prior treatment, but its synthesis is relatively expensive.

10 However, uric acid is the main nitrogen excretion product of reptiles, birds and certain 11 mammals. It is therefore readily available in poultry litter, in which it makes up to $55-63 \%$ of 12 total nitrogen and to $3 \%(\mathrm{w} / \mathrm{w})$ of the total weight (Nahm, 2003). Uric acid enters marine ecosystems in substantial amounts via faeces of seabirds. A recent study detected measurable effects of uric acid fertilisation in the vicinity of Great Cormorant (Phalacrocorax carbo sinensis) colonies in the Baltic Sea (Gagnon et al., 2013). More than 50\% of the nitrogen taken up by algae at the observed sites originated from faecal uric acid deposition. While it is obvious that valuable nitrogen sources such as uric acid are quickly taken up by marine food webs, the mechanisms and microbial uric acid utilization and the microorganisms involved in this process remain largely unknown (Capone et al., 2009) Trials in sediments and soil revealed that microbes related to Acinetobacter baumannii can use uric acid as a nitrogen source during hydrocarbon degradation (Koren et al., 2003). When uric acid was applied to marine sediments in form of guano, microbes related to the genera Alcanivorax, Halomonas and Alteromonas could be enriched and isolated (Knezevich et al., 2006). Uric acid is an intermediate of the purine metabolism and is oxidised by uricase enzymes. Members of the Bacillus, Pseudomonas, Alcaligenes and Microbacterium genera have been identified as being able to use uric acid as carbon and nitrogen source (Koren et al., 2003; Vogels \& van der Drift, 1976). It is therefore highly probable that marine oil degradation with uric acid as main nitrogen source requires a consortium of currently unknown uric acid-degrading microbes and highly adapted HCBs. This knowledge gap is very surprising as uric acid-based bioremediation approaches have been investigated in trials at different scales (Koren et al., 2003; Knezevich et al., 2006; Nikopoulou, 2008, 2013).

32 This study aimed at the identification of marine microbial consortia members involved in the metabolism of uric acid in marine oil-degrading and uric acid utilizing consortia. 
Study sites

The sampling sites were located along the Northern and Southern shores of the Mediterranean Sea and the Red Sea. In particular: (1) the site of El Max (31 $\left.{ }^{\circ} 9^{\prime} 31.20^{\prime \prime N}, 29^{\circ} 50^{\prime} 28.20^{\prime \prime} \mathrm{E}\right)$ located west of the city of Alexandria, Egypt, the El Max seashore is the most contaminated in the Alexandria area exceeding the legal limits for heavy metals, PAHs and other crude oilderived pollutants; (2) the Bizerte lagoon $\left(37^{\circ} 16^{\prime} 7.72^{\prime \prime} \mathrm{N}, 9^{\circ} 53^{\prime} 19.61^{\prime \prime}\right.$ E) in the North of Tunisia, is polluted by petroleum components in the area adjacent to STIR refinery, the Tunisian Company for Refining Industries; (3) the Jordanian coast in the Gulf of Aqaba $\left(30^{\circ} 22^{\prime} 42^{\prime}\right.$ 'N, $\left.25^{\circ} 24^{\prime} 57^{\prime \prime} \mathrm{E}\right)$ in the north-west end of the Red Sea. The Gulf of Aqaba is the northernmost tropical marine ecosystem but hosts an oil terminal that yearly moves up to 30 million tons and is subjected to accidental oil spills at the oil terminal as well as spills (with high sulphur concentrations) during loading and bunkering operations at the industrial jetty; (4) the harbour of Ancona, Italy, $\left(43^{\circ} 37^{\prime} \mathrm{N} ; 13^{\circ} 30^{\prime} 15^{\prime \prime} \mathrm{E}\right)$, which is a major ferry terminal and industrial port on the Adriatic Sea and heavily contaminated with PAHs and heavy metals.

Microcosm design and set-up

Two different sizes of microcosms were set up for this trial (Table 1). For the analysis of microbial population dynamics, respiration and other parameters, a series of 16 microcosm flasks (two parallels each for a uric acid and an ammonium treatment for sediments from the Ancona, Aqaba, Bizerte and El Max sites, respectively) was set up in $500 \mathrm{~mL}$ Erlenmeyer flasks (Microcosms 1-8). Seventy-five grams of sand, $5 \mathrm{~mL}$ of crude oil and $150 \mathrm{~mL}$ of the ONR7 medium mentioned above were used. Overall nutrient concentrations and C:N:P ratios were identical to the ones in the large microcosms mentioned above. All microcosms were incubated at $20^{\circ} \mathrm{C}$ for 28 days in a shaking incubator at $120 \mathrm{rpm}$. The small microcosms were sampled on days $0,2,4,6,8,10,12,15,18,21,24$ and 28 . On these days, $1 \mathrm{~mL}$ of sediment was taken for DNA extraction, $1 \mathrm{~mL}$ of supernatant was taken for photometric measurements and $6 \mathrm{ml}$ were taken for emulsification measurements. Fifty millilitres of each microcosm were taken on days $0,4,8,12,15,18,21,24$ and 28 to measure respiration activity. The sample volume taken for each sampling day was replaced by an equivalent volume of modified ONR7a medium. For ammonium treatments, the equivalent amount of ammonium chloride and disodium hydrogen phosphate was added to compensate for loss of water-soluble 
1 insoluble in water, sample volumes were replaced with ONR7a containing disodium

2 hydrogen phosphate but no ammonium chloride.

3 Four control treatments with a total of eight control flasks were set up. A negative control 4 contained only sterile sand and ONR7a. Two further controls contained sand, ONR 7a, crude oil and either uric acid or ammonium chloride solution but no sediment sample. Finally, one control contained oil, sterile sand, ONR7a medium and a sediment sample from Ancona harbour, but no additional nitrogen source or phosphorus source was provided (Microcosms 9 $-12)$.

To generate high DNA yields required for sequencing analysis, a series of up-scaled microcosms was set up (Microcosms 13-20). 1 L Erlenmeyer flasks were filled with $150 \mathrm{~g}$ of sand (Sigma-Aldrich, St. Louis, USA), sterilized and spiked with $10 \mathrm{~mL}$ of sterile filtered Arabian light crude oil. One gram of sediment from each sampling site was mixed into the oil-spiked sand as the inoculum. For the Aqaba site, $2 \mathrm{~g}$ of sediment were used, as this sediment was very coarse. Three hundred millilitres of modified ONR7a medium (Dyksterhouse et al., 1995) (omitting ammonium chloride and disodium hydrogen phosphate) were added. We added $5 \mathrm{ml}$ of Arabian light crude oil, which based upon average literature values for density and molecular weight equals about $300 \mathrm{mM}$ of $\mathrm{C}$ (Wang et al., 2003), 5 $\mathrm{mM}$ of $\mathrm{NH}_{4} \mathrm{Cl}$ and $0.5 \mathrm{mM}$ of $\mathrm{Na}_{2} \mathrm{HPO}_{4}$ resulting in a molar $\mathrm{N}: \mathrm{P}$ ratio of approximately 10:1. For each uric acid treatment microcosm (UA), $0.21 \mathrm{~g}(1.25 \mathrm{mmol}=5 \mathrm{mmol} \mathrm{N})$ of uric acid was provided as nitrogen source while the ammonium treatment microcosms (NP) were each supplied with $2.5 \mathrm{~mL}$ of a $2 \mathrm{M}$ ammonium chloride solution (5 mmol; $\mathrm{pH} 7.8$ ). Both treatments also contained $2.5 \mathrm{ml}$ of a $0.2 \mathrm{M}$ disodium hydrogen phosphate solution $(0.5 \mathrm{mmol}$; $\mathrm{pH}$ 7.8). Excess amounts of crude oil were added to compensate for the $35 \%$ carbon losses due to evaporation of volatile hydrocarbons over the course of the experiment (Wang et al., 2003). Including losses due to evaporation the $\mathrm{C} / \mathrm{N} / \mathrm{P}$ ratio was approximately 400:10:1. One hundred and four millilitres of mesocosm water were replaced by an equal volume of modified ONR 7containing uric acid or ammonium as described above on days $0,4,8,12,15$ and 18. The microcosms were destructively sampled on day 21 .

\section{Emulsification measurements}

33 Six millilitres of sample were pipetted into screw cap glass centrifuge tubes $(15 \times 150 \mathrm{~mm})$. 34 Six millilitres of $n$-hexane (Sigma-Aldrich, St. Louis, USA) were added and each tube was 35 vortex-shaken at maximum rate for $60 \mathrm{~s}$. The resulting emulsion was left to settle for $24 \mathrm{~h}$ at 36 room temperature and the height of the interface which settled between hexane and the aqueous phase was measured with a calliper. 
Oxygen consumption was measured using a Micro-Oxymax Respirometer (Columbus Instruments, Columbus, OH, USA) to serve as a proxy for total aerobic metabolic activity. Fifty millilitre aliquots were transferred to $100 \mathrm{~mL}$ screw top glass bottles (VWR, Lutterworth, UK) and connected to the respirometer. Automatic volume determination was performed for each glass bottle prior to respiration measurement. Measurements were performed every $2 \mathrm{~h}$ for a total of $72 \mathrm{~h}$. For each measurement, sensors and bottles were automatically purged with sterile air to avoid inhibitory effects of oxygen depletion.

\section{Photometric determination of ammonium, nitrate and protein concentration}

Due to the presence of emulsified oil in most microcosms, all samples underwent a pretreatment before photometric measurement. Briefly, each $1 \mathrm{~mL}$ sample was sonicated using a Soniprep 150 sonicator (MSE, London, UK) for $20 \mathrm{~s}$ in an ice bath. Subsequently, samples were centrifuged at 16,000g in an Eppendorf centrifuge to separate biomass incorporating oil at the bottom of the centrifuge tube from free oil at the surface of the aqueous phase. Sub samples were taken from the centre of the aqueous phase, diluted and applied in photometric analysis. A nitroprusside-based assay was used (Mulvaney et al., 1996) for the determination of ammonium concentrations and a vanadium chloride based method (Miranda et al., 2001) was used to measure nitrate concentration. Protein concentrations were determined as a proxy for microbial growth using the BioRad Protein assay (BioRad, Carlsbad, USA) according to the manufacturer's guidelines.

Isolation, 16S rRNA gene sequencing and phylogenetic analysis of uric acid-utilising microorganisms

31 Samples of one hundred microliters were taken from microcosms 13 to 20 on days 6 and 12 32 of the experiment. A serial dilution was performed using sterile modified ONR7a medium that contained neither nitrogen nor phosphorus source. $100 \mu \mathrm{L}$ of each dilution were plated on modified ONR7a agar plates containing $0.3 \%(\mathrm{w} / \mathrm{v})$ uric acid and $0.45 \mathrm{mM}$ disodium hydrogen phosphate. Due to its low solubility in water, uric acid forms a precipitate, which makes the agar plates appear milky. The plates were incubated at $20^{\circ} \mathrm{C}$ for up to $5 \mathrm{~d}$. Isolates 
1 colonies. Eight colonies of each variant were re-plated twice on modified ONR7a agar plates

2 to obtain pure isolates. Each isolate was tested for the ability to use uric acid as the sole

3 source of carbon and nitrogen in $15 \mathrm{~mL}$ polypropylene tubes containing $5 \mathrm{ml}$ ONR7 liquid medium supplemented with uric acid and disodium hydrogen phosphate, as described above. Tubes were incubated at $20^{\circ} \mathrm{C}$ for 5 to $7 \mathrm{~d}$. Colony PCR of each isolate was performed using primers F530 and R1492 to amplify the 16S rRNA gene. Each $20 \mu \mathrm{L}$ PCR reaction contained $2 \mu \mathrm{L}$ of PCR Buffer B (Roboklon, Berlin, Germany), $2.5 \mathrm{mM} \mathrm{MgCl}_{2}, 200 \mu \mathrm{M}$ of each dNTP (Promega, Madison, WI), 10 nM each of primer F530 (5'- GTG CCA GCM GCC GCG G-3') and primer 1492r (5'- GGT TAC CTT GTT ACG ACT T-3'), 0.5 U of Taq DNA Polymerase (Roboklon, Berlin, Germany) and $4 \mu \mathrm{L}$ of a PCR-enhancing mixture (3 M betaine and $1 \%$ 11 Tween 20). PCR products were visualised on $1 \%$ agarose gels. Sequencing was performed at 12 Macrogen (Amsterdam, Netherlands) with primers F530 and R1492. The processing and assembly of DNA sequences as well as testing for chimeric sequences was conducted using BioEdit as described before (Ashelford et al., 2005; Gertler et al., 2012). Sequence alignment was performed against a database of validly published reference DNA sequences of typed strains using ClustalW (Larkin et al., 2007). The sequences were clustered by maximum parsimony according to the recommendation of the MODELTEST software (Posada \& Crandall, 1998) and phylogenetic tree was constructed using MEGA 4.0 as previously described (Gertler et al., 2010, 2012). DNA sequences of Halomonas isolates were submitted to the European Nucleotide Archive (ENA) under the accession numbers HG803097 to HG803140.

\section{Nucleic acid extraction}

As both Illumina sequencing and RISA performed in this study require different qualities and quantities of DNA, nucleic acids were isolated using two approaches. For Illumina sequencing (Microcosms 13-20), a modified CTAB-phenol-chloroform extraction was performed. Briefly, for the initial cell lysis, $20 \mathrm{~g}$ of sediment, $1 \mathrm{~g}$ of 2-3 mm glass beads (5 to 6 glass beads) and $10 \mathrm{mg}$ of lysozyme were placed in a $50 \mathrm{~mL}$ centrifuge tube. Fifteen millilitres of extraction buffer containing $10 \%(\mathrm{w} / \mathrm{v})$ sucrose, $20 \%(\mathrm{v} / \mathrm{v})$ modified CTAB solution (5\% CTAB; $0.35 \mathrm{M} \mathrm{NaCl}, 120 \mathrm{mM} \mathrm{Na}_{2} \mathrm{HPO}_{4} \mathrm{pH} 8.0$; to improve solubilisation of $\mathrm{CTAB}$, this solution is a $1: 1$ mixture of $10 \% \mathrm{CTAB} / 0.7 \mathrm{M} \mathrm{NaCl}$ and $240 \mathrm{mM} \mathrm{Na} \mathrm{HPO}_{4}$ solutions), $100 \mathrm{mM}$ NaEDTA pH 8.0 and $100 \mathrm{mM}$ Tris/HCl pH 8.0 were added to the sample, and all tubes were incubated on a shaker at $220 \mathrm{rpm}$ and $30^{\circ} \mathrm{C}$ for at least $30 \mathrm{~min}$. Twenty milligrams of proteinase $\mathrm{K}$ were added to each tube, and all samples were incubated on a shaker at $220 \mathrm{rpm}$ and $50^{\circ} \mathrm{C}$ for at least $30 \mathrm{~min}$. Two millilitres of a $20 \%$ SDS (w/v) solution were added and all samples were incubated on a shaker at $100 \mathrm{rpm}$ and $60^{\circ} \mathrm{C}$ for $20 \mathrm{~min}$. 
1 incubated on a shaker at $100 \mathrm{rpm}$ and $60^{\circ} \mathrm{C}$ for $30 \mathrm{~min}$. Resulting mixtures were centrifuged 2 at $17,000 \mathrm{~g}$ for $10 \mathrm{~min}$.

3 Resulting supernatants were transferred to another $50 \mathrm{~mL}$ centrifuge tube. The pellets were extracted again by adding $5 \mathrm{~mL}$ of extraction buffer to the pellet, mixing for $5 \mathrm{~min}$ and centrifugation as described above. The resulting supernatant was combined with the supernatant from the previous step. To remove organic contaminants, the combined supernatants were treated with $0.5 \mathrm{~g}$ of activated charcoal, which was subsequently removed by centrifugation at $20,000 \mathrm{~g}$ for $10 \mathrm{~min}$. Supernatants were transferred to new $50 \mathrm{~mL}$ centrifuge tubes and 0.5 M NaEDTA solution was added to the supernatants for receive a final EDTA concentration of $2.5 \mathrm{mM}$. Deproteination and DNA extraction were performed by adding one supernatant volume of phenol/chloroform/isoamylalcohol (PCI) solution (25:24:1 (v/v); Sigma-Aldrich, St. Louis, USA). The mixtures of PCI and supernatant were inverted manually for $2 \mathrm{~min}$ and subsequently centrifuged at $3,700 \mathrm{~g}$ for $10 \mathrm{~min}$. The aqueous phase was transferred to a new centrifuge tube and extracted again with chloroform/isoamylalcohol (24:1 (v/v); Sigma-Aldrich, St. Louis, USA). The resulting supernatants were precipitated by adding 0.8 volumes of isopropanol and incubating at $4^{\circ} \mathrm{C}$ overnight. Precipitates were collected by centrifugation at $18,000 \mathrm{rcf}$ for $20 \mathrm{~min}$ at $4^{\circ} \mathrm{C}$. Pellets were washed once with $70 \%$ ethanol and centrifuged at $18,000 \mathrm{rcf}$ for $10 \mathrm{~min}$. The supernatants were discarded, and remaining traces of ethanol were evaporated by air-drying. The pellets were dissolved in 100 $\mu \mathrm{L}$ of sterile deionised water. Nucleic acid extraction for RISA (Microcosms 1-12) was performed with a similar but less time-consuming and less waste-producing protocol due to the large amount of samples. Briefly, a phenol-chloroform extraction (Anderson \& MacKay, 1983) was used with an additional bead-beating step using one sterile $2 \mathrm{~mm}$ glass bead and bead beating at a setting of 4.0 for $20 \mathrm{~s}$ in a FP120 Cell Disruptor (Qbiogene, Carlsbad, USA) prior to the addition of SDS buffer.

\section{PCR, Ribosomal Intergenic Spacer Analysis}

PCR amplification of the intergenic spacers was performed using the primer set ITSF/ITSReub (Cardinale et al., 2004). Each $20 \mu \mathrm{L}$ reaction contained $2.5 \mathrm{mM} \mathrm{MgCl}, 200$ $\mu \mathrm{M}$ of each dNTP (Promega, Madison, WI), $10 \mathrm{nM}$ each of primer ITSF (5'- TCG TAA CAA GGT AGC CGT A-3') and ITSReub (5'- GCC AAG GCA TCC ACC-3') (Cardinale et al., 2004), $2 \mu \mathrm{L}$ of PCR Buffer B (Roboklon, Berlin, Germany), $0.5 \mathrm{U}$ of Taq DNA Polymerase (Roboklon, Berlin, Germany) and $4 \mu \mathrm{L}$ of a PCR-enhancing mixture (3 $\mathrm{M}$ betaine and $1 \%$

36 Tween 20). One nanogram of template DNA was used in each reaction. PCR was performed 
1 (BioRad, Hercules, USA). Gel electrophoresis was conducted on an Ingeny PhorU gel 2 electrophoresis apparatus using 1x TAE 6\% acrylamide gels with $1 \mathrm{x}$ TAE buffer at $110 \mathrm{~V}$ for $316 \mathrm{~h}$. The gels were stained with $40 \mathrm{~mL}$ 1x SYBR Gold (Life Technologies, Carlsbad, USA) 4 staining solution and visualised on a ChemiDoc XRS gel documentation system (BioRad, Hercules, USA).

\section{RISA fingerprinting analysis, multivariate statistical analysis}

Quantity One software (BioRad, Hercules, USA) was used for band identification and band matching, with a total of 112 band classes assigned. Following square root transformation, principal coordinate analysis was conducted with PAST using the Bray-Curtis dissimilarity (Hammer et al., 2001). Analysis of similarities (ANOSIM) was performed using PRIMER 6 (Clarke and Gorley, 2006). One-way ANOSIMs of the whole data set were conducted for the parameters "Location (Inoculum)", "Nitrogen source" and "Sampling time". To investigate effects of different nitrogen sources on individual subsets of the data for each location, a twoway nested ANOSIM was conducted.

DNA sequencing, assembly, gene prediction and annotation

18 Sequencing was performed by pair-end sequencing (Illumina Hiseq 2000) at BGI - Beijing 19 Genomics Insititute (Hong Kong, People's Republic of China). A total of 20,000,000 sequences with a mean read length of 170 nts were obtained per sample. For gene prediction, the software MetaGeneMark (version 2.10, default parameters; Noguchi et al., 2006) was used to predict the open reading frames (ORFs) based on the assembly results. The predicted amino acid sequences were then aligned using the KEGG database (Kanehisa et al., 1997, 2004, 2006) through BLAST (version 2.2.23), and the relevant information was extracted and summarised with self-developed scripts. For Illumina Hiseq 2000 sequencing and data processing, the DNA samples were sequenced following standard pipelines of the Illumina platform. Data filtration was conducted with the following in-house scripts (1) Removal of reads with $3 \mathrm{~N}$ bases and removal of reads contaminated by adapters (15 bases overlapped by reads and adapter), (2) removal of reads with 20 bp low quality (20) bases and (3) removal of duplication contamination. The read removal process is simultaneously conducted for the read1 and read2 operation. The resulting data set can be used for subsequent analysis of quality data (Clean Data) (Li et al., 2010). SOAPdenovo (Version 1.0, http://soap.genomics.org.cn/soapdenovo.html) was used for the assembly of Illumina Hiseq 2000 sequences and to assemble filtered data. Assembly results with the best $\mathrm{N}_{50}$ contig length were optimized by in-house scripts (Li et al., 2008).

Rarefaction curves of the observed species were established for each sample to analyse the species sampling coverage. Known bacteria, fungi and archaea sequences were extracted 
1 from the Nucleotide database by an in-house script. Filtered reads were mapped to these 2 sequences by SOAPaligner (version 2.21) ( $\mathrm{Li}$ et al., 2009). Mapped reads were classified in 3 different taxonomic levels (including domain, phylum, class, order, family, genus, and species) and corresponding abundance was also calculated by in-house scripts. All rarefraction curves (data not shown) indicated closeness to saturation in each of the samples, which suggests that biases during the comparative analysis within the metagenomes herein reported were not introduced due to differences in microbial coverage.

Accession numbers

10 The projects have been registered as umbrella BioProjects at NCBI with the IDs 11 PRJNA222667 [for MGS-AQ(UA)], PRJNA222664 [for MGS-ANC(UA)], PRJNA222665 12 [for MGS-BIZ(AMM)], PRJNA222666 [for MGS-EIMAX(UA)], PRJNA222663 [for MGS13 ANC(AMM)]. These Whole Genome Shotgun projects have been deposited at $14 \mathrm{DDBJ} / \mathrm{EMBL} / \mathrm{GenBank}$ under the accession numbers AZIG00000000 [for MGS-AQ(UA)], 15 AZIH00000000 [for MGS-ANC(UA)], AZII00000000 [for MGS-BIZ(AMM)], 16 AZIJ00000000 [for MGS-EIMAX(UA)] and AZIK00000000 [for MGS-ANC(AMM)]. The 17 versions described in this paper are versions AZIG0100000 [for MGS-AQ(UA)], 18 AZIH0100000 [for MGS-ANC(UA)], AZII0100000 [for MGS-BIZ(AMM)], AZIJ0100000 19 [for MGS-EIMAX(UA)] and AZIK0100000 [for MGS-ANC(AMM)]. Abbreviations used for 20 database deposition as follows: MGS, MetaGenome Sequence; AQ(UA), Aqaba uric acid 21 (microcosm 16); ANC(UA), Ancona uric acid (microcosm 14); ElMAX(UA), El Max uric acid (microcosm 18); ANC(AMM), Ancona ammonium (microcosm 13); BIZ(AMM), Bizerte ammonium (microcosm 19).

\section{Results}

\section{Microbial metabolic activity in microcosms}

All microcosms showed a strong increase in respiration rate and emulsification immediately after the start of the experiment corresponding to high aerobic microbial activity (Fig.1). However, total rates of respiration for all uric acid treatments were 8-fold higher at its peak compared to the initial values. These values were higher than the peak values for the ammonium treatments, which, in turn, were 5-fold higher than the initial values. The peak values for respiration were reached on day 12 in the uric acid-containing microcosms $(2,4,6$,

36 8) and between days 8 and 15 in the ammonium-containing microcosms $(1,3,5,7)$.

37 Following their respective peaks, respiration dropped to base levels after day 18 in uric acid 
1 treatments but remained elevated in the Ammonium treatments due to a continuous influx of 2 ammonium during sampling procedures. The analysis of total respiration showed that uric 3 acid treatments had, on average, a 1.5-fold higher total respiration $\left(620 \mathrm{mg} / \mathrm{L} \mathrm{O} \mathrm{O}_{2}\right)$ than ammonium treatments $\left(376 \mathrm{mg} / \mathrm{L} \mathrm{O}_{2}\right)$.

The emulsification assay served as a proxy for the activity of hydrocarbon degrading microbes. Emulsification occurred in both treatments after day 4 of the experiment and peaked at day 10 in both cases. Following the maximum, emulsification dropped to $50 \%$ of the peak value but remained constant until the end of the experiment. The error bars for all curves are relatively high, which can be explained by temporal shifts in metabolic activity in individual microcosms by \pm 2 days.

11 The photometric quantitation of ammonium and protein concentrations shows an anticyclic 12 trend in both treatments. Ammonium becomes readily available at high concentrations in both the uric acid and ammonium treatments. The ammonium concentration increased immediately after the start of the experiment (with ammonium values not detectable in uric acid treatments at day 0 of the trial) and peaked at day 4 . Following the peak, ammonium concentrations rapidly decreased from day 6 to 18 in both microcosms and remained low in uric acid treatments in contrast to NP microcosm which were resupplied with ammonium during sampling procedures. Small residual amounts of ammonium could be detected in both treatments at the end of the experiment.

20 Conversely, protein concentrations, which served as a proxy of total biomass, increased in 21 both treatments from day 6 of the experiment onward. However, the concentrations dropped after day 18 in all uric acid treatments but increased constantly in Ammonium treatments.

\section{Dynamic changes in microbial community composition}

Microbial community changes and composition were analysed using Ribosomal Intergenic Spacer Analysis (RISA) and Pair-end sequencing. Figure 2 shows the results of the Principal Coordinate Analysis (PCoA). The individual fingerprints used for this analysis are presented in the Supplementary Figure 2. The PCoA plot shows no distinctively separated clusters, indicating that the microbial communities in the microcosms are essentially similar over the course of the experiment. This similarity can be explained by the choice of samples, which come from sites with a legacy of hydrocarbon pollution as well as from the same body of water and climate zone. The data points for both the NP and UA treatments overlap for all sampling sites, indicating that the treatments ultimately produce identical microbial communities. This result is supported by the ANOSIM results presented in Supplementary Table 1a. 
1 Statistically significant similarities could be detected when analysing the factor "nitrogen 2 source". High similarities (R value -0.156) were detected when using two-way ANOSIM to 3 investigate the similarities between the UA and NP treatments for samples at each location. 4 Data points from individual locations, on the other hand, show subtle differences separating the Ancona and Aqaba samples, although there is some degree of congruence for the Northern African samples; however, the ANOSIM analysis did not indicate significant similarities between the microbial communities from individual locations (Supplementary Table 1b). Most obvious is the separation of the early sampling points (days $0-10$ ) in the upper right section of Figure 2 from the bulk of the late sampling points (days $12-28$ ). This trend coincides with the peaks of both emulsification (days $10-12$ in both treatments) and protein 11 production (days 12 -20), which is strongly supported by the ANOSIM results presented in 12 Supplementary Table 1c. Significant similarities could be detected in the RISA profiles for 13 samples taken between days 4 and 8 , as well as for samples taken between days 12 to 28 .

Metagenome analysis of marine oil-degrading consortia: bacterial diversity and composition DNA isolated from each microbial community sampled from microcosms 13-20 at the day 21 was sequenced using Illumina HiSeq 2000. The data and annotation features resulting from the assemblies of each sample are shown in Supplementary Table 2. Three samples from uric acid enrichments and two samples from ammonium enrichments were chosen to ensure that all sampling sites were covered and to allow a direct comparison of at least one site. The samples for sequencing were selected based upon the results of RISA and whether the ANOSIM analysis showed high similarities between the ammonium and uric acid treatments for a particular site, omitting redundant samples. However, to confirm these similarities, both treatments of the Ancona microcosms were analysed. The results of this analysis, presented in Figure 3, show compositional similarities between the uric acid and ammonium treatments of the Ancona sample when the 16S rRNA partial gene sequences obtained in the non-assembled Illumina reads were surveyed. For this purpose, we used only sequences with a length of $>100$ nucleotides. Similarities could be observed between the uric acid treatments of the Ancona and El Max site (microcosms 14, 18), as well as between the ammonium treatments of the Ancona and Bizerte sites (microcosms 13, 19). The Aqaba uric acid treatment (microcosm 16) showed relatively little similarity to any other treatments and was characterised by a high percentage of Pseudomonadaceae. Supplementary Table 3 indicates that Pseudomonas spp. were the most abundant microbes in every treatment. They were more abundant in the uric acid treatments, and their percentage increased along a North-South gradient for these treatments. Specialised hydrocarbon-degrading microbes belonging to the genera Alcanivorax, Cycloclasticus, Oleiphilus, Oleispira and Thalassolituus were detected in all treatments. Most prominent among these microbes were the Alcanivorax spp., which were 
1 more abundant after NP (from 4.99 to $16.51 \%$ total reads) rather than uric acid treatments

2 (from 0.44 to $3.94 \%$ total reads). The analysis of the metagenomic data showed that most

3 Alcanivorax-like DNA sequences belonged to Alcanivorax borkumensis.

In parallel to this result, the ammonium treatments contained a higher percentage ( $\sim 9.0$-fold increment) of Alphaproteobacteria, consisting mostly of Roseovarius and Sulfitobacter spp. Conversely, uric acid treatments were more likely to contain Marinobacter spp. (up to 2.0fold) and Halomonas spp. (up to 3.8 -fold). Direct comparison of both Ancona treatments showed a 1.5-fold higher percentage of Halomonas spp. and a 2-fold higher percentage of Marinobacter spp. but an 8-fold lower abundance of Alphaproteobacteria in the uric acid treatments. The percentage of Pseudomonas spp. and Alcanivorax spp., however, remained similarly high.

\section{Genomic signatures associated with uric acid metabolism.}

In the metagenomes we identified 176,914 potential protein-coding genes (cut-off of $\geq 20$ amino acid-long sequences), with El Max containing the highest number $(61,277)$. Functional assignment of the predicted genes was made on the basis of BLASTP analysis against a reference dataset for Clusters of Orthologous Groups (COGs) and Kyoto Encyclopedia of Genes and Genomes (KEGG) assignments. Circa 63.1-67.9\% could be assigned to $\mathrm{COG}$, and 54.0-63.7\% could be assigned to KEGG pathways (Supplementary Table 2). On average, 6.6 to 12.8 genes belonged to $\mathrm{COG}$ and from 5.6 to 11.2 genes belonged to KEGG, depending on the sample.

All annotated data sets were examined for gene homologs involved in uric acid utilisation pathways. In both eukaryotes and prokaryotes, two urate hydroxylases (EC 1.7.3.3 and EC 1.14.13.113) have previously been shown to convert uric acid into 5-hydroxyisourate, which is ultimately broken down into urea and finally into ammonium. However, no genes with similarity to genes coding for either of these two enzymes could be detected in any of the datasets. In contrast, genes coding for enzymes that catalyse downstream reactions of uric acid utilisation, including allantoinase, allantoicase, allantoate amidohydrolase, ureidoglycolate hydrolase, 5-hydroxyisourate hydrolase, and 2-oxo-4-hydroxy-4-carboxy-5ureidoimidazoline (OHCU) decarboxylases, could be found (Supplementary Table 4a - e). The percentages of genes involved in uric acid metabolism were up to 3 -fold higher in UA enrichments; they were particularly enriched ( $\sim 0.11 \%$ protein-coding genes) in Aqaba uric acid treatments while being particularly depleted in the Bizerte ammonium treatments $(0.02 \%$ total protein-coding genes). However, we note that the NP-treated microcosm from Ancona 
1 port also contained a significant amount of those genes $(0.06 \%$ total protein-coding genes.

2 Binning analysis by BLASTP search further suggests that at least 15 (for Aqaba - uric acid),

311 (for Ancona - ammonium), 14 (for Ancona - uric acid), 2 (for Bizerte - ammonium) and

415 (for El Max- uric acid) distinct microorganisms may be potentially involved in the metabolism of uric acid and/or its degradation products. A direct comparison between the Ancona ammonium and uric acid treatments revealed that only 4 out of 25 of such microorganisms were shared, suggesting that most of the uric acid-utilization microbes are distinct to those using ammonium. The possibility that the microbes in the Ancona ammonium enrichment possess genes encoding pathways for uric acid utilization could not be ruled out; however, the data presented in this study suggests that in the presence of uric acid such proteins do not support bacterial growth and thus under these conditions other microbes are enriched. Supplementary Table $4 \mathrm{a}$ - e show genes for uric acid catabolism with similarity to the genes found in members of Aeromonadaceae, Halomonadaceae, Pseudomonaceae and Rhodobactereaceae within the three metagenomes from the uric acid enrichments, whereas the metagenomes from the ammonium enrichments predominantly contained uric acid catabolism genes from Alteromonadaceae, Oceanospirillaceae and Rhodobacteraceae. To further investigate which of these potential degrading organisms may play a role in uric acid metabolism, a cultivation-approach was used.

\section{Isolation of uric acid-degrading bacteria}

Uric acid-degrading bacteria were picked based upon the formation of a halo around the colony and purified to a single colony by triple repetitive plating on the same type of agar. As mentioned above, all isolates were successfully grown in ONR7a liquid media containing uric acid as sole source of nitrogen and carbon.

The 16S rRNA gene sequencing results (Supplementary Table 5) revealed that 44 of the 47 isolates belonged to the genus Halomonas. Two further isolates from the Bizerte enrichment belonged to the genus Marinobacter, and one isolate from the El Max enrichment was identified as a member of the genus Pelomonas. Phylogenetic analysis (Figure 4) showed that isolates were closely related to Halomonas alkaliphila, $H$. cupida, $H$. salfondinae, $H$. titanicae, $H$. venusta and $H$. ventosae. With a few exceptions, the majority of the isolates showed location specificity. Most of the isolates from Ancona were closely related to $H$. alkaliphila; however, the isolates from Aqaba were predominately related to $H$. venusta and $H$. hydrothermalis. The majority of isolates from the Bizerte enrichments clustered with $H$. salfodinae and $H$. pacifica. Finally, all but two isolates from the El Max/Alexandria enrichments were distantly related to $H$. cupida and $H$. shangliensis. 
1 The analysis of the metagenomic data mentioned above showed that genes with a high 2 similarity to genetic material from Halomonas spp. were present in all five metagenomes, 3 albeit in low percentages, ranging from $1.4 \%$ to $6.1 \%$ as revealed by abundance of SSU 4 rRNA signatures. The percentage of Halomonas-like sequences was elevated in the uric acid 5 enrichments.

6

7

8 
Discussion:

3 Comparison of uric acid and ammonium as nitrogen source in sediment microcosms

4 Ammonium concentrations were measured as a proxy for nitrogen uptake in ammonium treatments and uric acid utilization in uric acid treatments, as it was the sole nitrogen source in the ammonium treatments but also an intermediate of uric acid conversion in the uric acid treatments. The ammonium treatments did show a relatively low ammonium concentration at the beginning of the experiment with a subsequent increase. This result indicates a general problem with ammonium and phosphate additions to seawater. As seawater is a saline solution, the addition of further ions may lead to precipitation. The addition of phosphate to artificial seawater leads to precipitation of calcium or magnesium ammonium phosphate or phosphates of trace elements that could affect microbial growth (Atlas, 1975). In this study, phosphate was added to artificial seawater that contained Fe and other trace elements that precipitate with both ammonium and phosphate (e.g. as a ferrous ammonium phosphate). The secondary increase of ammonium concentrations could be explained by consumption of both iron and phosphate for microbial growth and a subsequent release of ammonium into the medium.

18 The ammonium concentrations in the uric acid treatments increased rapidly to approximately $270 \mathrm{mg} / \mathrm{L}$, which corresponds to $80 \%$ of the total uric acid nitrogen introduced into the microcosms. As uric acid was the only nitrogen-containing substance in these microcosms, it stands to reason that it is quickly and almost entirely converted into ammonium. Photometric measurements of nitrate showed no significant increase in its concentrations (data not shown) in either treatment throughout the course of the experiment; it can therefore be assumed that uric acid was entirely transformed into ammonium. Several recent studies have focused on the use of uric acid as a stand-alone nitrogen source or as a bioremediation strategy in combination with other substances. Koren and co-authors (2003) described the use of pure uric acid in hydrocarbon-polluted sediments and succeeded in isolating a strain of Acinetobacter baumannii that directly used uric acid for hydrocarbon degradation. In a further study, Knezevich et al. (2006) described the application of guano as a complex source of uric acid and a soluble nitrogen source for bioremediation using both $A$. borkumensis and mixed oil-degrading microbial communities in an open system. While the uric acid-fed communities showed rapid growth, with cell numbers of up to $10^{8}$ cells $/ \mathrm{mL}$ and degradation of $70 \%$ of total oil hydrocarbons, the cell numbers in the NPK-fertilised experiment remained two orders of magnitude lower and oil degradation was negligible. The data presented in our study is in concordance with a number of previous publications

36 (Knezevich et al., 2006; Nikolopoulou and Kalogerakis, 2008; Nikolopoulou et al., 2013) 
1 treatments. Microbial communities grew more rapidly and developed cell numbers three 2 orders of magnitude higher than comparable treatments with soluble nitrogen sources 3 (Nikolopolou et al., 2013). Furthermore, aliphatic hydrocarbon degradation and PAH 4 degradation were both significantly accelerated in presence of uric acid (Nikolopoulou and Kalogerakis, 2008; Nikolopoulou et al., 2013). Comparison of growth rates furthermore indicated that addition of molasses and rhamnolipids may speed up the microbial growth in the experiment, but is not stringently required for oil-degrading microbes to reach comparable cell numbers (Nikopoulou and Kalogerakis, 2008).

\section{Microbial population dynamics of microbial communities utilising uric acid}

11 Two recent studies have investigated the use of fertilisers containing uric acid by oil12 degrading microbial communities (Knezevich et al., 2006; Nikopoulou et al., 2013). The 13 findings from both studies indicate the presence of Alcanivorax borkumensis in enrichments 14 or microcosms supplied with uric acid. In the study of Knezevich et al. (2006), Alcanivorax spp., Alteromonas and Halomonas spp. were enriched and isolated. The results of our study also show abundance of Alcanivorax spp. in all samples. However, all uric acid enrichments showed a significantly lower abundance of these microbes in uric acid enrichments compared to ammonium enrichments. Despite the extensive research into the Alcanivorax genus, only one strain able to use uric acid as nitrogen source has been described to date (Knezevich et al., 2006). The data from our study suggests that the degradation of uric acid could be performed by other members of the microbial consortium present in uric acid-amended hydrocarbon spiked enrichments. Uric acid enrichment also showed a high conversion rate into ammonium and elevated respiration rates in contrast to ammonium enrichment from days 0 to 9 . However, the use of uric acid from days 4 to 6 did not lead to an increase in protein or biomass production. This result could be due to the extremely low $\mathrm{CN}$ ratio of uric acid, which limits the total amount of biomass microbes can produce when grown on uric acid alone. Figures $1 \mathrm{a}$ and $1 \mathrm{c}$ show that both emulsification and protein production begin to increase from day 6 onward. At this time, large quantities of uric acid have already been turned over into ammonium.

30 Therefore, microbial hydrocarbon degradation using uric acid as a nitrogen source could be 31 described as a two-stage process involving the primary conversion of uric acid into ammonium and a secondary stage in which marine hydrocarbonoclastic microbes use ammonium as the primary nitrogen source.

This two-stage process can also be observed in Figure 2, as most DNA fingerprints converged in a large cluster towards the end of the experiment. Similar trends have been observed in previous studies with similar experimental conditions (Gertler et al., 2009a,b; 2012). 
1 Microbial climax communities with high similarity formed within 14 days of the experiment,

2 yet a subtle location specificity of the communities can be observed. While these differences

3 can be observed within the DNA fingerprints from Aqaba, Ancona, Bizerte, and El Max

4 (Figure S1), they are not statistically significant, as shown by multivariate analysis. The results of ANOSIM analysis indicated that the microbial communities showed significant similarities at two stages during the experiment, within days 4 to 8 and from day 12 to the end of the experiment, supporting the hypothesis of a two-stage process. The formation of a highly similar climax community within 14 to 21 days in different locations has been observed in a previous study involving the Irish Sea, the North Sea and the Mediterranean

10 (Gertler et al., 2012). While obligate marine oil-degrading bacteria, most notably Alcanivorax 11 species, form the backbone and most active hydrocarbon degraders of these consortia (Gertler 12 et al., 2009a,b), there is a certain degree of variability in the composition of other consortium 13 members. In contrast to the intense clustering, the data points from days 0 to 6 of the 14 experiment are located outside of this cluster, indicating a community change. The ANOSIM results (Supplementary Table 1c) indicate that within this period a highly similar community that differs from the oil-degrading climax community established after day 12 formed in all microcosms.

18 Metagenome sequencing was conducted for five samples from Aqaba, Ancona, Bizerte and El 19 Max (Microcosms 13, 14, 16, 18, 19), which were selected after the RISA fingerprinting. One 20 sample from each Aqaba, Bizerte and El Max was chosen to cover location-specific 21 characteristics of the sediment, while two samples from Ancona (one for the uric acid and one for the ammonium treatment) were selected to directly compare the effects of both treatments in one location. The sample material was based upon single biological replicate to preserve maximum coverage and sequencing depth as well as for other technical reasons. This procedure however does not take in account possible culture variability. A solution to this problem could be pooling of replicate samples, which reduce this bias, it would also reduce the overall coverage. Multiplexing of individual samples in a single sequencing run on the other hand bears the risk of adapter misidentification while true replication of samples (e.g. for a time series of microcosm samples) is still financially prohibitive. To ensure the validity of metagenome data in this study, rarefraction curves were established for each of the metagenome data sets and that were close to saturation and exhibited low standard deviation. Metagenome sequencing confirmed the composition of the microbial consortia to be dominated by Pseudomonas spp. and to some extent by Alcanivorax spp. in all cases. Pseudomonas spp. were the most abundant species in all consortia, while the Ancona - uric acid, Ancona - ammonium and Bizerte - ammonium samples contained 4 - 16\% of

36 Alcanivorax spp. -related 16S rRNA reads in the whole SSU rRNA dataset. The samples 37 from El Max and Aqaba showed an overall similar species composition to the other samples, 
1 but a significantly higher percentage of Pseudomonas spp.. This data is only apparently in 2 contrast to previous studies conducted with similar microcosms that showed a significantly 3 higher percentage of Alcanivorax spp. (Gertler et al., 2010a, 2012) considering that the 4 current study used the sediment rather than seawater as the inoculum. Furthermore, the 5 genomes of Pseudomonas spp. are known to possess six or more rRNA operons, in contrast to 6 the three rRNA operons present in the genome of Alcanivorax borkumensis. This difference 7 in the number of operons could result in an overestimation of the numbers of pseudomonads 8 from 16S rRNA-related reads affiliated with Pseudomonas spp. in comparison to Alcanivorax 9 spp. Most notably, the composition of uric acid and ammonium-based enrichments from 10 Ancona proved to be similar, as both communities were enriched from identical inocula and 11 effectively supplied with ammonium, in the latter case directly added to the medium while in 12 the former case deriving from uric acid conversion. In addition to the clustering of uric acid 13 and ammonium enrichment fingerprints in Figure 2, these findings indicated that given 14 identical experimental parameters, uric acid supplementation could effectively produce 15 identical oil-degrading consortia due to the quick conversion of uric acid into ammonium. 16 This suggestion is statistically supported by nested two-way ANOSIM analysis of the RISA 17 location data sets. 
3 The analysis of the five metagenomes annotated within this study remained inconclusive regarding uric acid metabolism. Neither of the genomes showed any genes coding for uricase enzymes. However, urate oxidase activity has also been detected in other classes of enzymes, e.g., a laccase from a Lysobacter species (Tamaki et al., 2010). It is therefore possible that the actual genes responsible for urate oxidation in the analysed samples could not be detected because they were annotated differently. Genes coding for ureidoglycolate hydrolase (EC 5.3.19), 5-hydroxisoureate hydrolase (EC 3.5.2.17), allantoicase (EC 3.5.3.4.), allantoinase (EC 3.5.2.5) and OHCU decarboxylase (EC 4.1.1.-), on the other hand, were identified within the genomes. These genes are involved in uric acid metabolism and appeared three times more frequently in the metagenomes of the uric acid-based enrichments in relation to the total number of sequences. Furthermore, uric acid catabolism genes in ammonium-based enrichments were mostly similar to the genes of Oceanospirillaceae and Rhodobacteriaceae, whereas the uric acid catabolism genes in the uric acid-based enrichments were predominantly similar to genes found in members of Pseudomonadaceae and Halomonadaceae. The analysis of 16S rRNA data in the metagenomes showed comparable percentages of Halomonas spp. and Pseudomonas spp. in the uric acid treatments compared to ammonium treatments (Figure 3, Table S3). However, the direct comparison of the ammonium and uric acid treatments for the Ancona site revealed higher percentages of Halomonas spp. in the presence of uric acid and comparable percentages of Pseudomonas spp. in both treatments. The evidence from the metagenome analysis, therefore, indicates that the Halomonas spp. rather than the Pseudomonas spp. are the main utilisers of uric acid in this experiment.

In addition to the metagenome analysis, major degraders of uric acid were identified by cultivation and isolation at various points during the experiment. Ninety-three per cent of the isolates belonged to the genus Halomonas, a ubiquitous, oligotrophic, highly salt-tolerant and metabolically versatile group of microbes (Llamas et al., 2006; Mata et al., 2002). Halomonas strains capable of biopolymer and bioemulsifier production have been previously cultured (Gutierrez et al., 2013). Members of this genus with hydrocarbon-degradation capabilities have also been isolated (Mnif et al., 2009) and detected in oil-degrading consortia using culture-independent techniques (Kleinsteuber et al., 2006; Gutierrez et al., 2011). In contrast, the strains grown in this study were isolated in absence of hydrocarbons with uric acid as sole source of carbon, nitrogen and energy. While the results of this approach are subject to the

36 limitations of our cultivation techniques, the presence of similar Halomonas species is 
1 above. Sequences of Halomonas spp. made up between 1.4 and $6.1 \%$ of the total amount of 2 rRNA gene sequences.

3 The Halomonas isolates displayed a certain location specificity, with most isolates from each 4 sampling site clustering together independently of experiment time and nitrogen source. This clustering could be because the genus Halomonas is understudied, and relatively few genomes and DNA sequences of few isolates are present in the databases. Another possibility is that the metabolic versatility of this genus (Mata et al., 2002) may have lead to the selection of specific Halomonas strains with uricase activity adapted to the unique abiotic parameters at the specific sampling site, suggesting a biogeographic distribution of this genus.

\section{Prospects for uric acid application in bioremediation techniques}

This study investigates the population dynamics of oil-spiked marine sediment microcosms supplied with ammonium or uric acid in equal amounts and $\mathrm{C}: \mathrm{N}: \mathrm{P}$ ratios using identical crude oil batches. These procedures enable the direct comparison of microbial communities utilising either nitrogen source. The use of uric acid as a nitrogen source has been extensively discussed, experimented on and even patented, yet the mechanism of uric acid utilisation in an actual microbial community remains unknown. In our study, marine microbial communities dominated by Pseudomonas spp. and Alcanivorax spp., the most prominent, ubiquitous and competitive obligate hydrocarbon marine microbes, have been found. It was shown that uric acid could be quickly transformed into ammonium by members of the Halomonas genus. This observation was made for sediments originating from four independent sites located around the Mediterranean (Ancona, Bizerte, El Max) and the Red Sea (Aqaba), which implies that a similar pattern likely occurs on a more global scale.

As each uric acid molecule contains four nitrogen atoms, such a nitrogen source can be applied in lower molarities compared to ammonium. While problems with leaching can be successfully overcome by paraffin coating (urea) or the use of slow-release fertilisers (ammonium, nitrate) (Gertler et al., 2009), these treatments require industrial processes that greatly reduce the cost-efficiency of bioremediation. Uric acid, on the other hand, can be found in concentrations of $3-6 \%(\mathrm{w} / \mathrm{w})$ in poultry faeces, making up $60 \%$ of the total nitrogen. The global production of up to 50 billion chickens produces 50 million tons of faecal matter every year with an estimated content of 1.5 to 3 million tons of uric acid (Nahm, 2003). Poultry litter is currently used as an agricultural fertiliser after sterilisation through heat treatment and drying and are well established on the fertiliser market. This study has shown that uric acid can be quickly transformed into ammonium, which in turn is utilised by highly efficient oil-degrading microbes in marine sediments. This process was observed in sediment collected from various locations in different water bodies without the addition of allochtonous 
1 microbial communities, suggesting that cost-effective uric acid-based biostimulation

2 techniques can be successfully achieved in a wide range of marine sediments.

3

\section{Acknowledgements}

5 The authors would like to thank Anna Foster, Sarah Chesworth and Gordon Turner for their

6 help with photometric and respiration measurements. With exception of $\mathrm{XH}$ and JC, all

7 authors were supported by the FP7 Project ULIXES (FP7-KBBE-2010-266473). This work

8 was further funded by grant BIO2011-25012 from the Spanish Ministry of the

9 Economy and Competitiveness. FM was supported by Università degli Studi di Milano,

10 European Social Fund (FSE) and Regione Lombardia (contract "Dote Ricerca"). DD

11 acknowledges support of KAUST, King Abdullah University of Science and Technology. PG

12 acknowledges the support of the European Commission through the project Kill-Spill (FP7,

13 Contract Nr 312139). CG would like to thank Mr. Kyungsun Lee of Macrogen Inc. for his

14 courtesy regarding sequencing services, J. Cans, B. Strid and C. Hudson for continued advice

15 and inspiration as well as Delphine Lallias for her help with multivariate statistics and John

16 Flannery for proofreading this manuscript. 

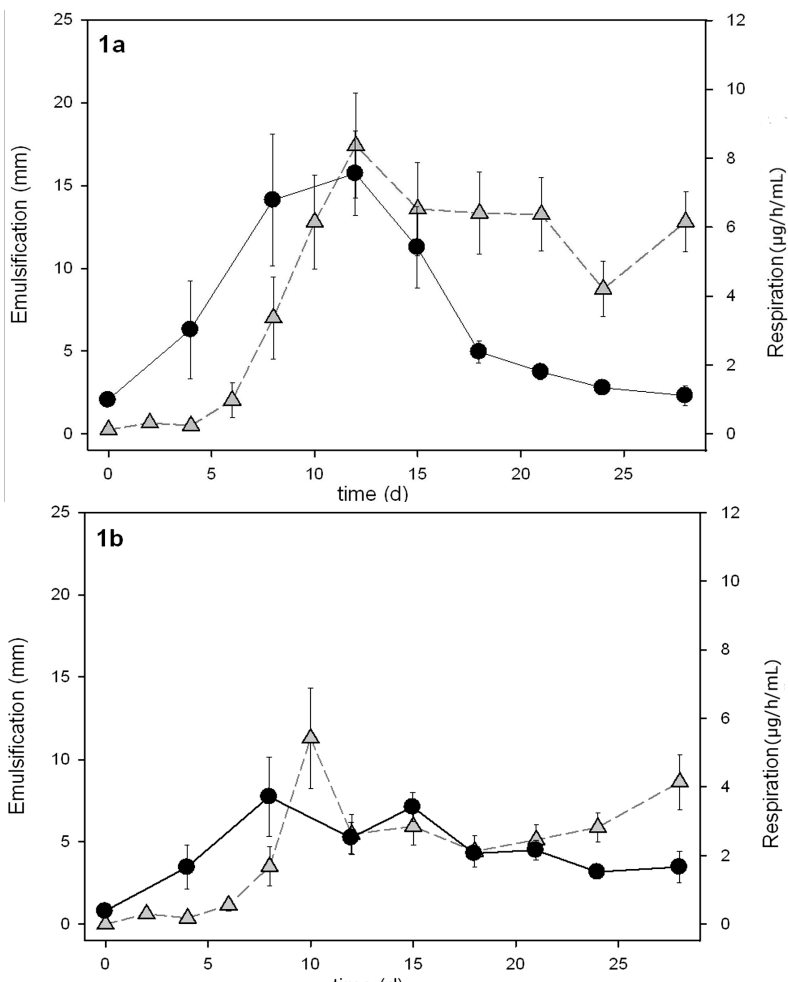

2

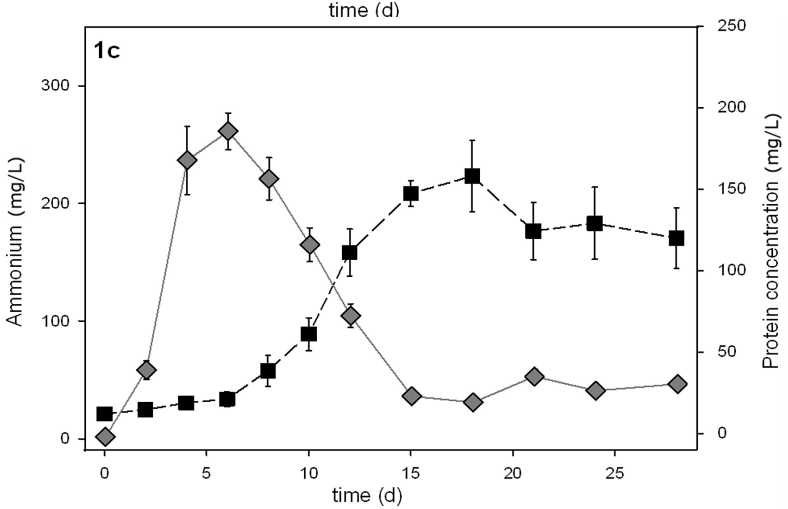

3

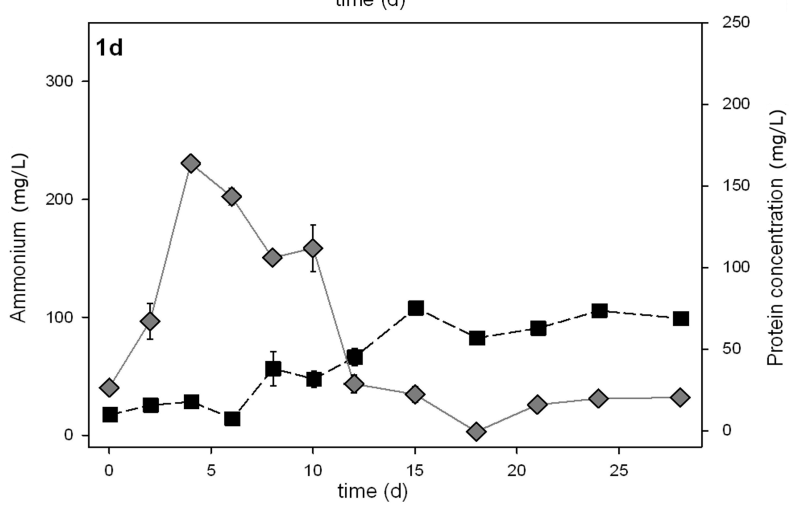

Figure 1a - d: Observations of protein and ammonium concentrations, and microbial respiration in microcosms. Figure 1a and $\mathbf{c}$ show data collected from uric acid enrichments (Microcosms 1, 3, 5, 7), and $\mathbf{1 b}$ and $\mathbf{d}$ from ammonium enrichments (Microcosms 2, 4, 6, 8). Filled circles represent respiration rates; open triangles indicate emulsification rates. Filled squares indicate total protein concentrations, whereas open diamonds show ammonium concentrations. All data points represent the mean of triplicates from each sampling point. Bars represent standard error. 


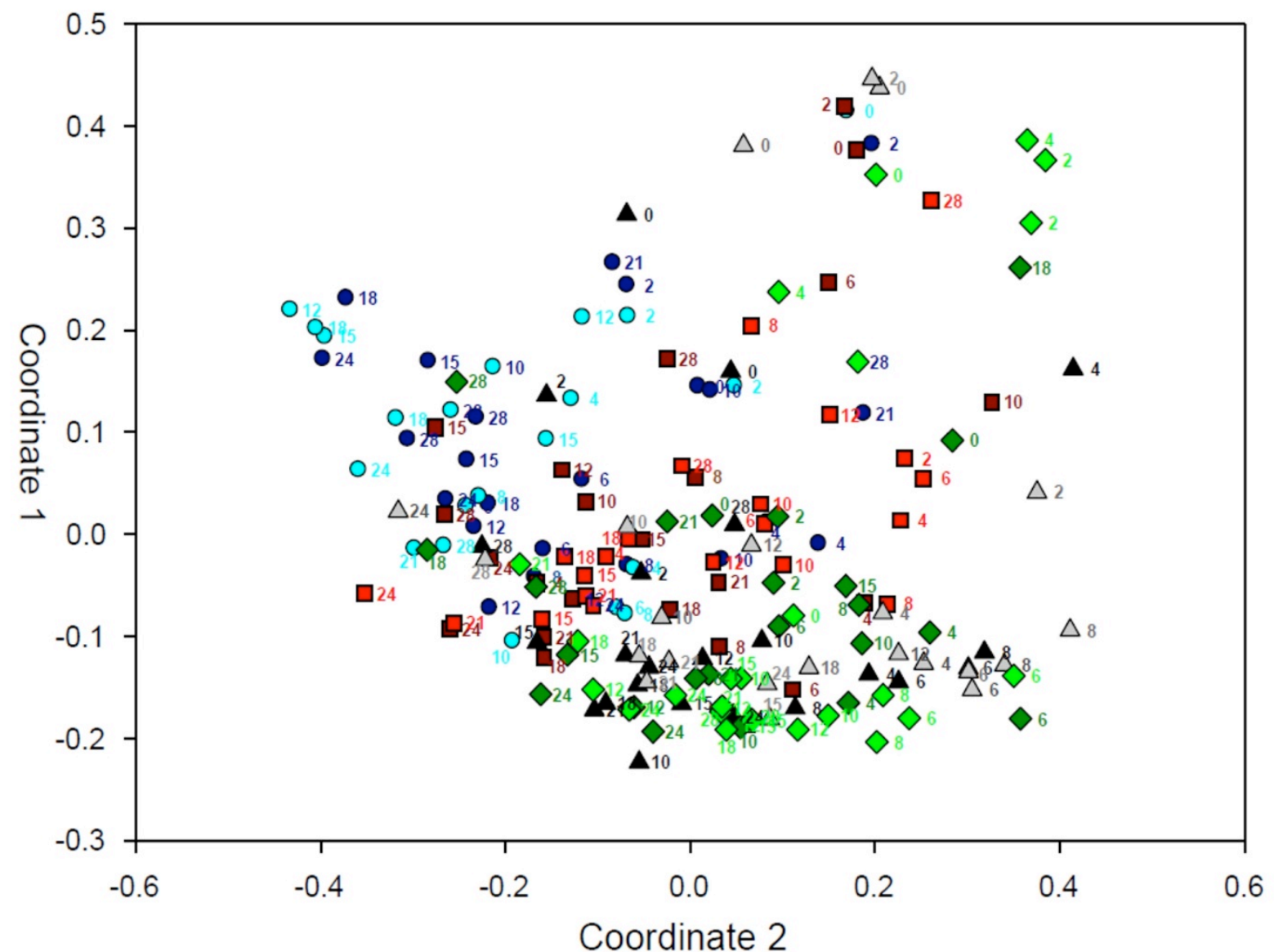

Figure 2: Principal coordinate Analysis of RISA fingerprints. Light colours indicate ammonium enrichments; dark colours indicate uric acid enrichments. Blue circles represent enrichments from Ancona, Italy (Microcosms 1, 2); red squares show samples from Aqaba, Jordan (Microcosms 3, 4). Grey triangles show samples from El Max, Alexandria, Egypt (Microcosms 5, 6), whereas green diamonds represent enrichments from Bizerte Lagoon, Tunisia (Microcosms 7, 8). Numbers correspond to the sampling day of each data point. 

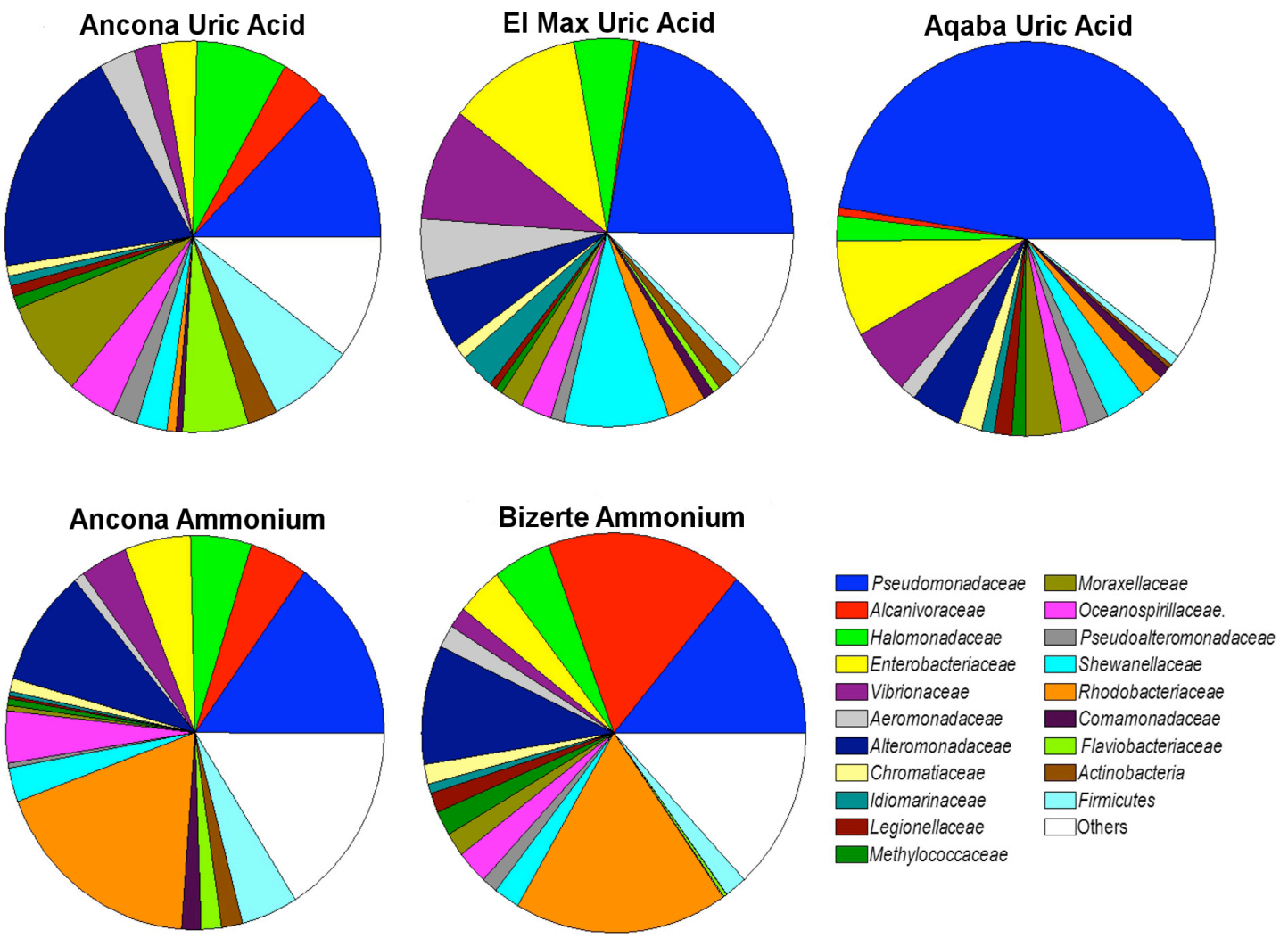

Figure 3: Relative abundances of microbial families within annotated metagenomes based on the $16 \mathrm{~S}$ small subunit rRNA data taken on day 21 from the following samples: Ancona Uric Acid (Microcosm 14), Ancona Ammonium (Microcosm 13), Bizerte Ammonium (Microcosm 19), Aqaba Uric Acid (Microcosm 16) and El Max Uric Acid (Microcosm 18). Only lineages with abundance of reads $>1 \%$ (SSU rRNA tags) are shown. Members of families belonging to Actinobacteria and Firmicutes were combined. 


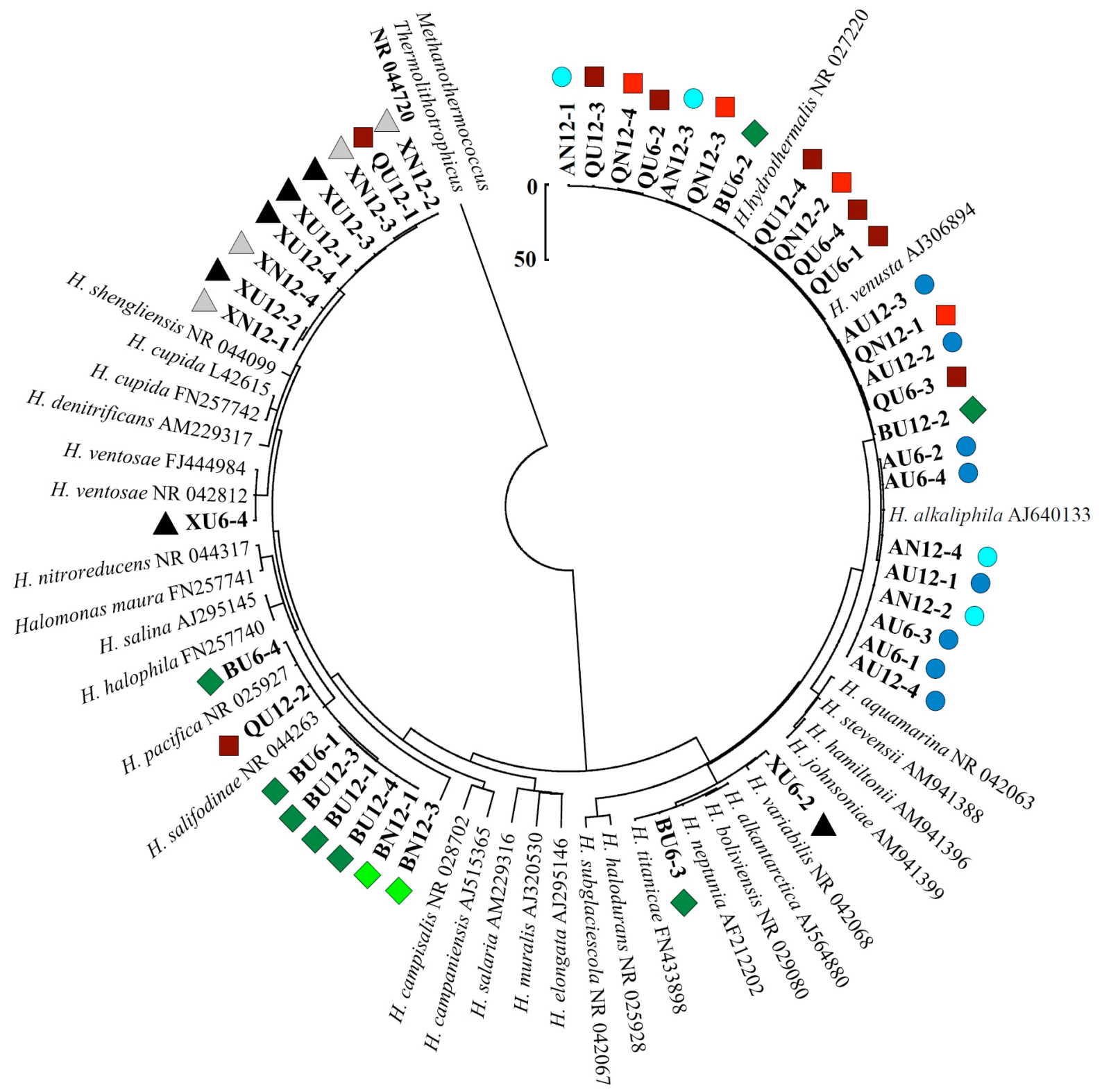

Figure 4: Rooted phylogenetic tree of 16S rRNA gene sequences from Halomonas spp. isolates grown on ONR7 agar containing uric acid. The prefix codes B (diamonds), X (triangles) Q (squares) and A (circles) represent the experimental sites Bizerte, El Max, Aqaba and Ancona, respectively. The prefix codes $\mathrm{U}$ and $\mathrm{N}$ represent the experimental treatment: 'UA or 'NP,' respectively. The colour coding of symbols corresponds to the colours used in Figure 2. Sequences were clustered by maximum parsimony according to the recommendation of the MODELTEST software (Posada \& Crandall, 1998). The 16S rRNA gene sequences of the most closely related sequences from type strains of Halomonas species were acquired from the NCBI nucleotide database. The evolutionary history was inferred using the Maximum Parsimony method (Eck \& Dayhoff, 1966). The bootstrap consensus tree inferred from 500 replicates is taken to represent the evolutionary history of the taxa analysed (Felsenstein, 1985. Branches corresponding to partitions reproduced in less than $50 \%$ bootstrap replicates are collapsed. The MP tree was obtained using the Close-Neighbor-Interchange algorithm (Nei \& Kumar, 2000) with search level 3 (Felsenstein, 1985; Nei \& Kumar, 2000) in which the initial trees were obtained with the random addition of sequences ( 10 replicates). The tree is drawn to scale, with branch lengths calculated using the average pathway method (Nei \& Kumar, 2000). Scale bar reflects the total nucleotide mismatch numbers in the whole sequence. All positions containing gaps and missing data were eliminated from the dataset (Complete Deletion option). There were a total of 952 positions in the final dataset, out of which 97 were parsimony informative. Phylogenetic analyses were conducted in MEGA4 (Tamura et al., 2007). 
1 Table 1: Composition of microcosms established in this study.

2

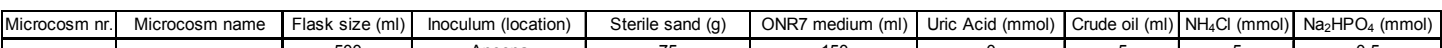

\begin{tabular}{|c|c|c|c|c|c|c|c|c|c|}
\hline & & & & & & & & & \\
\hline \multirow{2}{*}{1} & \multirow{2}{*}{ Ancona - ammonium } & 500 & Ancona & 75 & 150 & 0 & 5 & 5 & 0.5 \\
\hline & & 500 & Ancona & 75 & 150 & 0 & 5 & 5 & 0.5 \\
\hline \multirow{2}{*}{2} & \multirow{2}{*}{ Ancona - uric acid } & 500 & Ancona & 75 & 150 & 1.25 & 5 & 0 & 0.5 \\
\hline & & 500 & Ancona & 75 & 150 & 1.25 & 5 & 0 & 0.5 \\
\hline \multirow{2}{*}{3} & \multirow{2}{*}{ Aqaba - ammonium } & 500 & Aqaba & 75 & 150 & 0 & 5 & 5 & 0.5 \\
\hline & & 500 & Aqaba & 75 & 150 & 0 & 5 & 5 & 0.5 \\
\hline \multirow{2}{*}{4} & \multirow{2}{*}{ Aqaba - uric acid } & 500 & Aqaba & 75 & 150 & 1.25 & 5 & 0 & 0.5 \\
\hline & & 500 & Aqaba & 75 & 150 & 1.25 & 5 & 0 & 0.5 \\
\hline \multirow{2}{*}{5} & \multirow{2}{*}{ El Max - ammonium } & 500 & EI Max & 75 & 150 & 0 & 5 & 5 & 0.5 \\
\hline & & 500 & EI Max & 75 & 150 & 0 & 5 & 5 & 0.5 \\
\hline \multirow{2}{*}{6} & \multirow{2}{*}{ El Max - uric acid } & 500 & El Max & 75 & 150 & 1.25 & 5 & 0 & 0.5 \\
\hline & & 500 & EI Max & 75 & 150 & 1.25 & 5 & 0 & 0.5 \\
\hline \multirow{2}{*}{7} & \multirow{2}{*}{ Bizerta - ammonium } & 500 & Bizerta & 75 & 150 & 0 & 5 & 5 & 0.5 \\
\hline & & 500 & Bizerta & 75 & 150 & 0 & 5 & 5 & 0.5 \\
\hline \multirow{2}{*}{8} & \multirow{2}{*}{ Bizerta - uric acid } & 500 & Bizerta & 75 & 150 & 1.25 & 5 & 0 & 0.5 \\
\hline & & 500 & Bizerta & 75 & 150 & 1.25 & 5 & 0 & 0.5 \\
\hline \multirow{2}{*}{9} & \multirow{2}{*}{ Control - sterile } & 500 & none & 75 & 150 & 0 & 0 & 0 & 0 \\
\hline & & 500 & none & 75 & 150 & 0 & 0 & 0 & 0 \\
\hline \multirow{2}{*}{10} & \multirow{2}{*}{ Control - ammonium } & 500 & none & 75 & 150 & 0 & 5 & 5 & 0.5 \\
\hline & & 500 & none & 75 & 150 & 0 & 5 & 5 & 0.5 \\
\hline \multirow{2}{*}{11} & \multirow{2}{*}{ Control - uric acid } & 500 & none & 75 & 150 & 1.25 & 5 & 0 & 0.5 \\
\hline & & 500 & none & 75 & 150 & 1.25 & 5 & 0 & 0.5 \\
\hline \multirow{2}{*}{12} & \multirow{2}{*}{ Control - non sterile } & 500 & Ancona & 75 & 150 & 0 & 5 & 0 & 0 \\
\hline & & 500 & Ancona & 75 & 150 & 0 & 5 & 0 & 0 \\
\hline 13 & Ancona - ammonium & 1000 & Ancona & 150 & 300 & 2.5 & 10 & 10 & 1 \\
\hline 14 & \begin{tabular}{|l|} 
Ancona - uric acid \\
\end{tabular} & 1000 & Ancona & 150 & 300 & 2.5 & 10 & 10 & 1 \\
\hline 15 & Aqaba - ammonium & 1000 & Aqaba & 150 & 300 & 2.5 & 10 & 10 & 1 \\
\hline 16 & Aqaba - uric acid & 1000 & Aqaba & 150 & 300 & 2.5 & 10 & 10 & 1 \\
\hline 17 & \begin{tabular}{|l|} 
El Max - ammonium \\
\end{tabular} & 1000 & El Max & 150 & 300 & 2.5 & 10 & 10 & 1 \\
\hline 18 & \begin{tabular}{|l|} 
El Max - uric acid \\
\end{tabular} & 1000 & El Max & 150 & 300 & 2.5 & 10 & 10 & 1 \\
\hline 19 & Bizerta - ammonium & 1000 & Bizerta & 150 & 300 & 2.5 & 10 & 10 & 1 \\
\hline 20 & \begin{tabular}{|l|} 
Bizerta - uric acid \\
\end{tabular} & 1000 & Bizerta & 150 & 300 & 2.5 & 10 & 10 & 1 \\
\hline
\end{tabular}




\section{References}

Amirthanathan A \& Subramaniyan V (2012) Studies on uricase production by marine Bacillus cereus and its optimum conditions. Int J Med Biosci 1:5-12

Anderson DG \& McKay LL (1983) Simple and rapid method for isolating large plasmid DNA from lactic streptococci. Appl Environ Microbiol 46:549-552

Ashelford KE, Chuzhanova NA, Fry JC, Jones AJ \& Weightman AJ (2005) At least 1 in 20, 16S rRNA sequence records currently held in public repositories is estimated to contain substantial anomalies. Appl Environ Microbiol 71: 7724-7736.

Atlas EL (1975) Phosphate equilibria in seawater and interstitial waters. PhD thesis. Oregon State Univ., Corvallis, Oregon.

Atlas RM \& Bartha R (1972) Degradation and mineralization of petroleum by two bacteria isolated from coastal waters. Biotechnol Bioeng 14:297-308

Capone DG, Bronk DA, Mulholland MR \& Carpenter EJ (2008) Nitrogen in the Marine Environment. (Academic Press, 2008). at http://www.sciencedirect.com/science/book/9780123725226.

Cardinale, M. et al. (2004) Comparison of different primer sets for use in automated ribosomal intergenic spacer analysis of complex bacterial communities. Appl Environ Microbiol 70:61476156

Clarke KR, Gorley RN (2006) PRIMER v6: User Manual/Tutorial. PRIMER-E, Plymouth.

Dyksterhouse SE, Gray JP, Herwig RP, Lara JC \& Staley JT (1995) Cycloclasticus pugetii gen. nov.,

sp. nov., an aromatic hydrocarbon-degrading bacterium from marine sediments. Int J Syst Bacteriol

45:116-123

Eck RV \& Dayhoff MO (1966) Atlas of Protein Sequence and Structure. National Biomedical

Research Foundation, Silver Springs, Maryland.

Fava F, Berselli S, Conte P, Piccolo A \& Marchetti, L (2004) Effects of humic substances and soya lecithin on the aerobic bioremediation of a soil historically contaminated by polycyclic aromatic hydrocarbons (PAHs). Biotechnol. Bioeng. 88:214-223

Felsenstein J (1985) Confidence limits on phylogenies: An approach using the bootstrap. Evolution 39:783-791

Gagnon K, Rothäusler E, Syrjänen A, Yli-Renko M \& Jormalainen V (2013) Seabird Fertilizes Baltic Sea Littoral Food Webs. PLoS ONE 8:e61284

Garcia HE, Locarnini RA, Boyer TP, Antonov JI, Zweng MM, Baranova OK \& Johnson DR (2010) World Ocean Atlas 2009, Volume 4: Nutrients (phosphate, nitrate, silicate). S. Levitus, Ed. NOAA Atlas NESDIS 71, U.S. Government Printing Office, Washington, D.C.

Gertler C, Gerdts G, Timmis KN, Yakimov MM \& Golyshin PN (2009a) Populations of heavy fuel oil-degrading marine microbial community in presence of oil sorbent materials. J Appl Microbiol. 107:590-605

Gertler C, Gerdts G, Timmis KN \& Golyshin PN (2009b) Microbial consortia in mesocosm bioremediation trial using oil sorbents, slow-release fertilizer and bioaugmentation. FEMS Microbiol. Ecol 69:288-300

Gertler C, Näther DJ, Cappello S, Gerdts G, Quilliam RS, Yakimov MM \& Golyshin PN (2012) Composition and dynamics of biostimulated indigenous oil-degrading microbial consortia from the Irish, North and Mediterranean Seas: a mesocosm study. FEMS Microbiol. Ecol 81:520-536

Gunkel, W (1967) Experimentell- ökologische Untersuchungen über die limitierenden Faktoren des mikrobiellen Ölabbaues im marinen Milieu. Helgol Wiss Meeresunters. 15:210-225

Gutierrez T, Singleton DR, Berry D, Yang T, Aitken MD \& Teske A (2013) Hydrocarbon-degrading bacteria enriched by the Deepwater Horizon oil spill identified by cultivation and DNA-SIP. ISME J 7:2091-2104

Gutierrez T, Berry D, Yang T, Mishamandani S, McKay L, Teske A \& Aitken MD (2013) Role of Bacterial Exopolysaccharides (EPS) in the Fate of the Oil Released during the Deepwater Horizon Oil Spill. PLoS ONE 8: e67717

Hammer Ø, Harper DAT \& Ryan PD (2001) PAST: palaeontological statistics software package for education and data analysis. Palaeontol Electronica 4:9

Hazen, T. C. Dubinsky EA, DeSantis TZ, Andersen GL, Piceno YM, Singh N, Jansson JK et al. (2010) Deep-Sea Oil Plume Enriches Indigenous Oil-Degrading Bacteria. Science 330:204-208

Head IM, Jones DM \& Röling WFM (2006) Marine microorganisms make a meal of oil. Nat Rev Microbiol 4:173-182

Jiménez N, Viñas M, Sabaté J, Díez S, Bayona JM, Solanas AM \& Albaiges J (2006) The Prestige Oil Spill. 2. Enhanced Biodegradation of a Heavy Fuel Oil under Field Conditions by the Use of an Oleophilic Fertilizer. Environ Sci Technol 40:2578-2585 
Kanehisa M, Goto S, Kawashima S, Okuno Y \& Hattori M (2004). The KEGG resource for deciphering the genome. Nucleic Acids Res 32:277-280

Kanehisa M (1997). A database for post-genome analysis. Trends Genet 13:375-376

Kanehisa M, Goto S, Hattori M, Aoki-Kinoshita KF, Itoh M, Kawashima S, et al. (2006). From genomics to chemical genomics: new developments in KEGG. Nucleic Acids Res 34: 354-357.

Kasai Y, Kishira H, Sasaki T, Syutsubo K, Watanabe K \& Harayama S (2002) Predominant growth of Alcanivorax strains in oil-contaminated and nutrient-supplemented sea water. Environ Microbiol 4:141-147

Kleinsteuber S, Riis V, Fetzer I, Harms H \& Müller S (2006) Population dynamics within a microbial consortium during growth on diesel fuel in saline environments. Appl Environ Microbiol 72:35313542

Knezevich V, Koren O, Ron EZ \& Rosenberg E (2006) Petroleum Bioremediation in Seawater Using Guano as the Fertilizer. Bioremed J 10:83-91

Koren O, Knezevic V, Ron EZ \& Rosenberg E (2003) Petroleum pollution bioremediation using waterinsoluble uric acid as the nitrogen source. Appl Environ Microbiol 69:6337-6339

Kube M, Chernikova TN, Al-Ramahi Y, Beloqui A, Lopez-Cortez N, Guazzaroni ME,

Heipieper HJ, Klages S, Kotsyurbenko OR, Langer I, Nechitaylo TY, Lünsdorf H, Fernández M, Juárez S, Ciordia S, Singer A, Kagan O, Egorova O, Petit PA, StogiosP, Kim Y, Tchigvintsev A, Flick R, Denaro R, Genovese M, Albar JP, Reva ON, Martínez-Gomariz M, Tran H, Ferrer M, Savchenko A, Yakunin AF, Yakimov MM, Golyshina OV, Reinhardt R \& Golyshin PN. (2013) Genome sequence and functional genomic analysis of the oil-degrading bacterium Oleispira antarctica. Nat Commun 4:2156Lai Q, Li W \& Shao Z (2012) Complete Genome Sequence of Alcanivorax dieselolei Type Strain B5. J Bact 194:6674-6674Larkin MA, Blackshields G, Brown NP, Chenna R, McGettigan PA, McWilliam H, Valentin F, Wallace IM, Wilm A, Lopez R, Thompson JD, Gibson TJ, Higgins DG (2007) Clustal W and Clustal X version 2.0. Bioinformatics 21: 2947-2948

Li R, Li Y, Kristiansen K, Wang J. (2008) SOAP: short oligonucleotide alignment program. Bioinformatics 24, 713-714.

Li R, Yu C, Li Y, Lam TW, Yiu SM, Kristiansen K, Wang J. (2009). SOAP2: an improved ultrafast tool for short read alignment. Bioinformatics 25:1966-1967

Li R, Zhu H, Ruan J, Qian W, Fang X, Shi Z, Li Y, Li S, Shan G, Kristiansen K, Li S, Yang H, Wang J, Wang J. (2010) De novo assembly of human genomes with massively parallel short read sequencing. Genome Res 20, 265-272)

Lee K, Tremblay GH \& Levy EM (1993) Bioremediation: Application Of Slow-Release Fertilizers On Low-Energy Shorelines. Internat Oil Spill Conf Proceed 1993:449-454

Lindstrom JE et al. (1991) Microbial populations and hydrocarbon biodegradation potentials in fertilized shoreline sediments affected by the T/V Exxon Valdez oil spill. Appl Environ Microbiol 57:2514-2522

Mata, JA, Martínez-Cánovas J, Quesada E \& Béjar VA (2002) Detailed phenotypic characterisation of the type strains of Halomonas species. Syst Appl Microbiol 25:360-375

Miranda KM, Espey MG \& Wink DA (2001) A rapid, simple spectrophotometric method for simultaneous detection of nitrate and nitrite. Nitric Oxide 5: 62-71

Mnif S, Chamkha M \& Sayadi S (2009) Isolation and characterization of Halomonas sp. strain C2SS100, a hydrocarbon-degrading bacterium under hypersaline conditions. J Appl Microbiol 107:785-794

Mulvaney, R. in Methods of soil analysis part 3: chemical methods. 1123-1184 (Soil Science Society of America, Inc., 1996).

Nahm KH (2003) Evaluation of the nitrogen content in poultry manure. World's Poultry Sci J 59:7788

Nei M \& Kumar S (2000) Molecular Evolution and Phylogenetics. Oxford University Press, New York.

Nikolopoulou M \& Kalogerakis N (2008) Enhanced bioremediation of crude oil utilizing lipophilic fertilizers combined with biosurfactants and molasses. Mar Poll Bull 56:1855-1861

Nikolopoulou M, Pasadakis N \& Kalogerakis N (2013) Evaluation of autochthonous bioaugmentation and biostimulation during microcosm-simulated oil spills. Mar Poll Bull 72:165-173

Noguchi H, Park J et al. (2006) MetaGene: prokaryotic gene finding from environmental genome shotgun sequences. Nucleic Acids Res 34:5623-5630

Posada D \& Crandall KA (1998) MODELTEST: testing the model of DNA substitution. Bioinformatics 14:817-818 
Sawada R, Ke R, Tsuji T, Sonoyama S \& Mitaku S (2007) Ratio of membrane proteins in total proteomes of prokaryota. Biophysics 3:37-45

Schneiker, S. et al. (2006) Genome sequence of the ubiquitous hydrocarbon-degrading marine bacterium Alcanivorax borkumensis. Nat Biotechnol 24:997-1004

Tamaki H, Matsuoka T, Yasuda Y, Hanada S, Kamagata Y, Nakamura K \& Sakasegawa S (2010) A novel laccase with urate oxidation activity from Lysobacter sp. T-15. J Biochem 148:481-489

Tamura K, Dudley J, Nei M \& Kumar S (2007) MEGA4: Molecular Evolutionary Genetics Analysis (MEGA) software version 4.0. Mol. Biol. Evol. 24:1596-1599

Vogels GD. \& Van der Drift C (1976) Degradation of purines and pyrimidines by microorganisms. Bacteriol Rev 40:403-468

Wang Z, Hollebone BP, Fingas M, Fieldhouse B, Sigouin L, Landriault M, SmithP, Noonan J \& Thouin G (2003) Characteristics of Spilled Oils, Fuels, and PetroleumProducts:1. Composition and Properties of Selected Oils. United States Environmental Protection Agency, National Exposure Research Laboratory, EPA/600/R-03/072

Wrenn BA, Zheng H, Kohar ES, Lee K \& Venosa AD (2001) Effect of Pulsed Additions of Nutrients on Oil Biodegradation in Continuous-Flow Beach Microcosms. Internat Oil Spill Conf Proceed 2001:339-344

Xu R, Lau ANL, Lim YG \& Obbard JP (2005) Bioremediation of oil-contaminated sediments on an inter-tidal shoreline using a slow-release fertilizer and chitosan. Mar Poll Bull 51:1062-1070

Yakimov MM, Gentile G, Bruni V, Cappello S, D'Auria G, Golyshin PN, Giuliano L (2004) Crude oilinduced structural shift of coastal bacterial communities of Rod Bay (Terra Nova Bay, Ross Sea, Antarctica) and characterization of cultured cold-adapted hydrocarbonoclastic bacteria. FEMS Microbiol Ecol 49:419-432

Yakimov MM, Timmis KN \& Golyshin PN (2007) Obligate oil-degrading marine bacteria. Curr Opin Biotechnol 18:257-266 

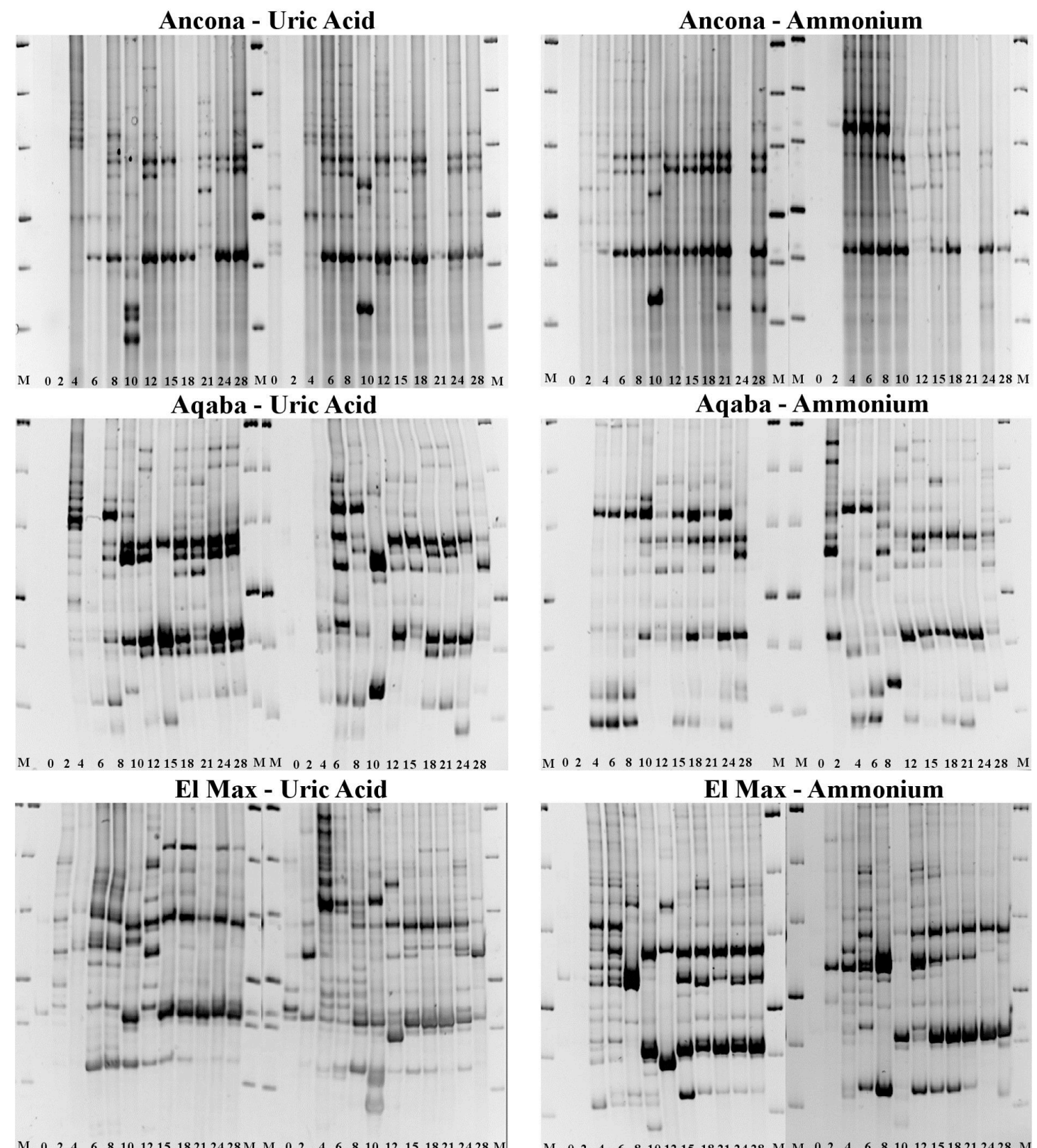

M $02446810121518212428 \quad M \quad M \quad 024468 \quad 121518212428$ M
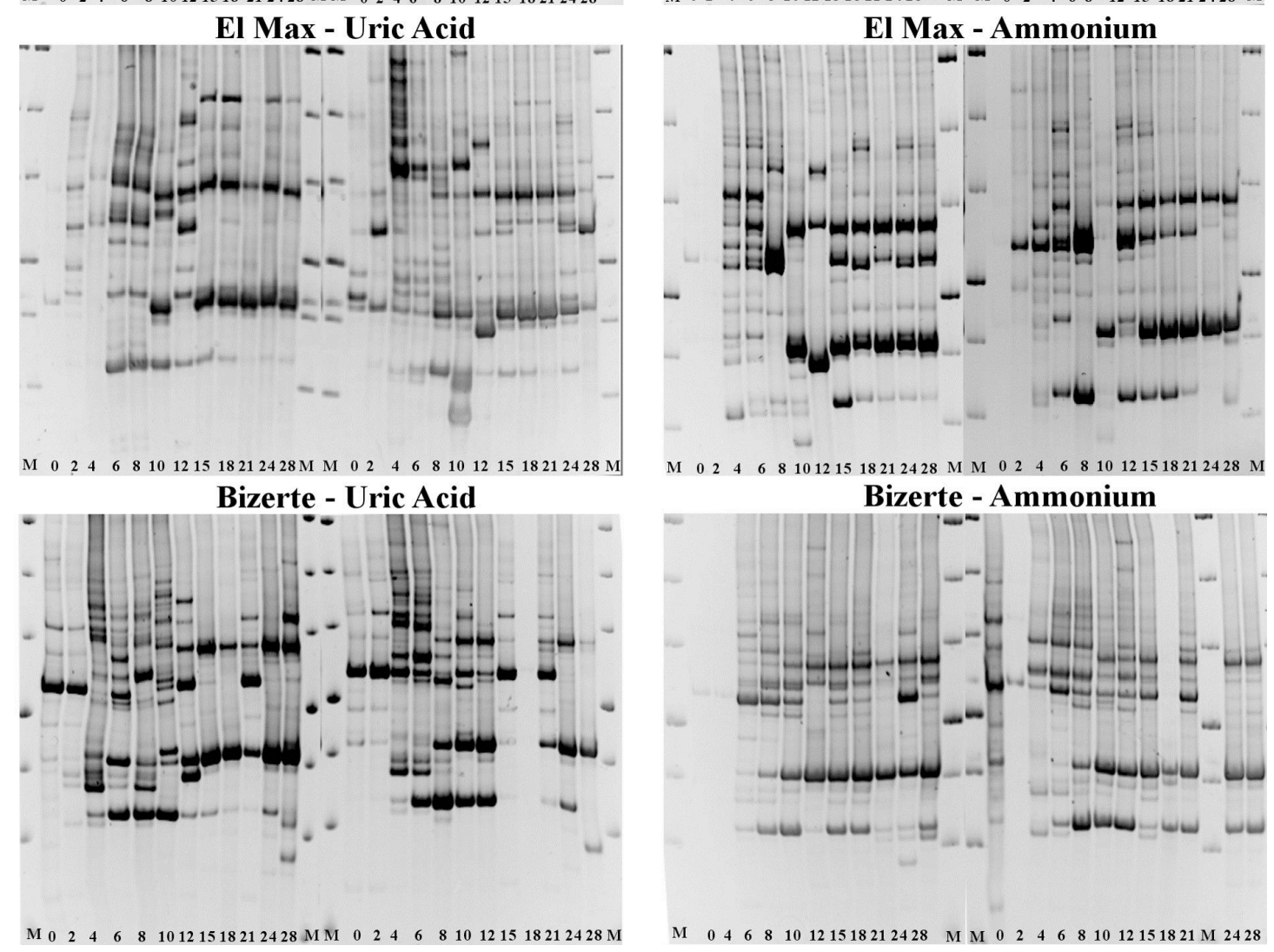

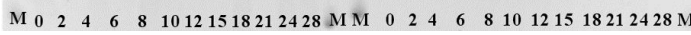

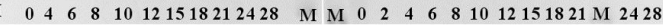

Supplementary Figure 1: RISA fingerprinting profiles used for the multivariate analysis presented in Figure 2 of the main text. Full DNA profiles of two out of three microcosm replicates were used to avoid mismatching in the band matching required for statistical analysis. All RISA fingerprints were run using the O'gene Ruler Plus (Thermo Scientific, Lutterworth, UK) as indicated by the letter M under the marker lanes. Gel images show DNA fragments of 300 to 5000 bp in length. Numbers under 
1 individual lanes represent sampling days. Fingerprints from microcosm replicates are displayed as 2 blocks of profiles with increasing sampling time (0 to 28 days). 
1 Supplementary Table 1a: ANOSIM results of bacterial community RISA fingerprinting profiles on 2 the basis of Bray-Curtis distance measure, showing the univariate partition of factors "Location", 3 "Nitrogen source" and "Sampling day" and the multivariate partition of factors "Location" within the 4 factor "Nitrogen source". The R-value was calculated based on the Null hypothesis of no similarity 5 between samples. The values highlighted in bold are statistically significant $(P<0.05)$.

\begin{tabular}{lll}
\hline One way ANOSIM & $R$ & Significance \% \\
\hline Location & 0.168 & 0.1 \\
Nitrogen source & $\mathbf{0 . 0 1 2}$ & $\mathbf{2 . 3}$ \\
Sampling day & 0.141 & 0.1 \\
\hline Nested two way ANOSIM & $R$ & Significance \% \\
\hline Location within N source & $\mathbf{- 0 . 1 5 6}$ & $\mathbf{8 5 . 7}$ \\
\hline
\end{tabular}

Supplementary Table 1b: $R$-values derived from ANOSIM pairwise comparisons of factor "(sampling) Location" values using Bray-Curtis values. The R-value was calculated based on the Null hypothesis of no similarity between samples. The values highlighted in bold are statistically significant

\begin{tabular}{lllll}
\hline & Ancona & Aqaba & Bizerte & El Max \\
\hline Ancona & & 0,164 & 0,25 & 0,254 \\
Aqaba & 0,164 & & 0,14 & 0,132 \\
Bizerte & 0,25 & 0,14 & & 0,076 \\
ElMax & 0,254 & 0,132 & 0,076 & \\
\hline
\end{tabular}

Supplementary Table 1c: $R$-values derived from ANOSIM pairwise comparisons of factor "Sampling 21 day" values using Bray-Curtis values. The R-value was calculated based on the Null hypothesis of no similarity between samples. The values highlighted in bold are statistically significant $(P<0.05)$.

\begin{tabular}{|c|c|c|c|c|c|c|c|c|c|c|c|c|}
\hline & 0 & 2 & 4 & 6 & 8 & 10 & 12 & 15 & 18 & 21 & 24 & 28 \\
\hline 0 & & -0.02 & 0.198 & 0.262 & 0.28 & 0.302 & 0.28 & 0.313 & 0.292 & 0.27 & 0.356 & 0.217 \\
\hline 2 & -0.02 & & 0.11 & 0.162 & 0.186 & 0.23 & 0.181 & 0.227 & 0.197 & 0.168 & 0.275 & 0.121 \\
\hline 4 & 0.198 & 0.11 & & -0.022 & 0.045 & 0.214 & 0.158 & 0.271 & 0.255 & 0.227 & 0.33 & 0.166 \\
\hline 6 & 0.262 & 0.162 & -0.022 & & -0.044 & 0.092 & 0.101 & 0.211 & 0.209 & 0.168 & 0.308 & 0.183 \\
\hline 8 & 0.28 & 0.186 & 0.045 & -0.044 & & 0.056 & 0.081 & 0.191 & 0.186 & 0.149 & 0.268 & 0.181 \\
\hline 10 & 0.302 & 0.23 & 0.214 & 0.092 & 0.056 & & 0.068 & 0.08 & 0.089 & 0.061 & 0.188 & 0.113 \\
\hline 12 & 0.28 & 0.181 & 0.158 & 0.101 & 0.081 & 0.068 & & 0.006 & -0.001 & -0.006 & 0.049 & 0.009 \\
\hline 15 & 0.313 & 0.227 & 0.271 & 0.211 & 0.191 & 0.08 & 0.006 & & -0.047 & -0.032 & -0.003 & -0.002 \\
\hline 18 & 0.292 & 0.197 & 0.255 & 0.209 & 0.186 & 0.089 & -0.001 & -0.047 & & -0.039 & -0.062 & -0.04 \\
\hline 21 & 0.27 & 0.168 & 0.227 & 0.168 & 0.149 & 0.061 & -0.006 & -0.032 & -0.039 & & -0.037 & -0.008 \\
\hline 24 & 0.356 & 0.275 & 0.33 & 0.308 & 0.268 & 0.188 & 0.049 & -0.003 & -0.062 & -0.037 & & 0.003 \\
\hline 28 & 0.217 & 0.121 & 0.166 & 0.183 & 0.181 & 0.113 & 0.009 & -0.002 & -0.04 & -0.008 & 0.003 & \\
\hline
\end{tabular}


2 Supplementary Table 2a: Data statistics for samples as obtained by Illumina sequencing

3

\begin{tabular}{|l|c|c|c|c|c|}
\hline Sample name & $\begin{array}{c}\text { Insert size } \\
(\mathrm{bp})\end{array}$ & $\begin{array}{c}\text { Sequence type } \\
(\mathrm{bp})\end{array}$ & $\begin{array}{c}\text { Raw reads } \\
(\mathrm{Mbp})\end{array}$ & $\begin{array}{c}\text { High quality } \\
\text { reads (Mbp) }\end{array}$ & $\begin{array}{c}\text { Clean data } \\
\text { rate (\%) }\end{array}$ \\
\hline MGS-AQ (UA) & 350 & Index 91 PE & 1,163 & 1,100 & 94.60 \\
\hline MGS-EIMAX (UA) & 350 & Index 91 PE & 1,149 & 1,100 & 95.73 \\
\hline MGS-ANC (UA) & 350 & Index 91 PE & 1,189 & 1,100 & 92.51 \\
\hline MGS-ANC (AMM) & 350 & Index 91 PE & 1,160 & 1,100 & 94.83 \\
\hline MGS-BIZ (AMM) & 350 & Index 91 PE & 1,266 & 1,100 & 86.88 \\
\hline
\end{tabular}

4

5 Supplementary Table 2b: Data statistics for the best assembly results

6

7

\begin{tabular}{|l|c|c|c|c|c|c|c|}
\hline Sample name & $\begin{array}{c}\text { Contig } \\
\text { num }\end{array}$ & $\begin{array}{c}\text { Contig lenght } \\
(\mathrm{bp})\end{array}$ & $\begin{array}{c}\text { N50 } \\
(\mathrm{bp})\end{array}$ & $\begin{array}{c}\text { N90 } \\
(\mathrm{bp})\end{array}$ & $\begin{array}{c}\text { Max } \\
(\mathrm{bp})\end{array}$ & $\begin{array}{c}\text { Min } \\
(\mathrm{bp})\end{array}$ & $\begin{array}{c}\text { Map to own } \\
\text { contigs (\%) }\end{array}$ \\
\hline MGS-AQ (UA) & 8,371 & $21,504,196$ & 12,101 & 753 & 273,210 & 500 & 83.38 \\
\hline MGS-ANC (AMM) & 12,469 & $24,752,834$ & 3,617 & 697 & 358,781 & 500 & 71.11 \\
\hline MGS-ANC (UA) & 12,691 & $19,364,101$ & 2,076 & 622 & 62,304 & 500 & 58.77 \\
\hline MGS-BIZ (AMM) & 12,814 & $20,253,283$ & 2,117 & 667 & 73,362 & 500 & 71.81 \\
\hline MGS-ElMAX (UA) & 23,220 & $45,489,734$ & 3,627 & 698 & 431,244 & 500 & 59.16 \\
\hline
\end{tabular}

8 Supplementary Table 2c: Gene prediction results

9

\begin{tabular}{|l|c|c|r|r|r|r}
\hline Sample name & ORFs & Avg. length (bp) & $\begin{array}{c}\text { Assigned } \\
\text { to COG }\end{array}$ & $\begin{array}{c}\text { Assigned to } \\
\text { KEGG }\end{array}$ & $\begin{array}{c}\text { Nr of } \\
\text { to COG }\end{array}$ & $\begin{array}{c}\text { Nr of } \\
\text { KEGG }\end{array}$ \\
\hline MGS-AQ (UA) & 26,866 & 712.98 & 18,242 & 17,111 & 2,719 & 2,780 \\
\hline MGS-ANC (AMM) & 32,180 & 690.62 & 21,101 & 19,029 & 2,855 & 2,856 \\
\hline MGS-ANC (UA) & 27,893 & 619.19 & 18,012 & 15,065 & 2,719 & 2,596 \\
\hline MGS-BIZ (AMM) & 28,698 & 619.65 & 18,261 & 15,796 & 2,433 & 2,790 \\
\hline MGS-ElMAX (UA) & 61,277 & 666.25 & 39,915 & 36,320 & 3,115 & 3,231 \\
\hline
\end{tabular}

10

11

12 
2 Supplementary Table 3: Percentages of microbial genera within metagenomes of samples from day 321 from the following microcosms: Ancona Uric Acid, Anacona Ammonium, Bizerte Ammonium, 4 Aqaba Uric Acid and El Max Uric Acid. The data are based on the frequency of the appearance of 5 reads of SSU rRNA. Genera were combined into families, classes or phyla where necessary.

6

\begin{tabular}{|c|c|c|c|c|c|c|}
\hline & & Bizerte NP & Ancona NP & Ancona UA & El Max UA & Aqaba UA \\
\hline \multirow{10}{*}{ Alphaproteobacteria } & Jannaschia spp. & 0,41 & 0,49 & 0,01 & 0,08 & 0,05 \\
\hline & Loktanella spp. & 0,71 & 0,84 & 0,04 & 0,13 & 0,10 \\
\hline & Oceanicola spp. & 0,48 & 0,65 & 0,03 & 0,13 & 0,05 \\
\hline & Paracoccus spp. & 0,80 & 0,62 & 0,01 & 0,28 & 0,12 \\
\hline & Rhodobacter spp. & 0,80 & 0,67 & 0,01 & 0,21 & 0,10 \\
\hline & Roseobacter spp. & 0,20 & 0,16 & 0,01 & 0,03 & 0,02 \\
\hline & Roseovarius spp. & 1,44 & 1,39 & 0,08 & 0,28 & 0,13 \\
\hline & Ruegeria spp. & 1,10 & 1,08 & 0,05 & 0,16 & 0,11 \\
\hline & Sulfitobacter spp. & 2,01 & 1,45 & 0,08 & 0,19 & 0,28 \\
\hline & Thalassospira spp. & 0,00 & 0,51 & 0,00 & 0,06 & 0,02 \\
\hline \multirow{3}{*}{ Betaproteobacteria } & Acidovorax spp. & 0,00 & 0,21 & 0,09 & 0,11 & 0,44 \\
\hline & Alcaligenes spp. & 0,01 & 0,00 & 0,00 & 0,05 & 0,14 \\
\hline & Polaromonas spp. & 0,00 & 0,08 & 0,04 & 0,05 & 0,12 \\
\hline \multirow{44}{*}{ Gammaproteobacteria } & Aeromonas spp. & 0,96 & 0,54 & 1,52 & 2,77 & 1,09 \\
\hline & Alcanivorax spp. & 16,35 & 4,94 & 3,94 & 0,35 & 0,64 \\
\hline & Acinetobacter spp. & 0,66 & 0,05 & 0,93 & 1,13 & 1,31 \\
\hline & Azotobacter spp. & 0,34 & 0,50 & 0,45 & 0,48 & 0,95 \\
\hline & Citrobacter spp. & 0,20 & 0,29 & 0,11 & 0,76 & 0,54 \\
\hline & Chromohalobacter spp. & 0,75 & 0,48 & 0,59 & 0,13 & 0,12 \\
\hline & Colvellia spp. & 0,19 & 0,23 & 0,11 & 0,44 & 0,28 \\
\hline & Cronobacter spp. & 0,14 & 0,00 & 0,07 & 0,70 & 0,48 \\
\hline & Cycloclasticus spp. & 0,52 & 0,01 & 0,04 & 0,01 & 0,00 \\
\hline & Enterobacter spp. & 0,31 & 0,42 & 0,17 & 0,70 & 0,48 \\
\hline & Gallibacterium spp. & 0,06 & 0,07 & 0,06 & 0,09 & 0,22 \\
\hline & Haemophilus spp. & 0,10 & 0,11 & 0,08 & 0,16 & 0,25 \\
\hline & Hahella spp. & 0,19 & 0,12 & 0,23 & 0,16 & 0,44 \\
\hline & Halomonas spp. & 3,64 & 4,18 & 6,05 & 4,18 & 1,40 \\
\hline & Idiomarina spp. & 0,68 & 0,26 & 0,76 & 2,67 & 0,97 \\
\hline & Legionella spp. & 1,59 & 0,23 & 0,91 & 0,63 & 1,52 \\
\hline & Marichromatium spp. & 0,18 & 0,15 & 0,14 & 0,16 & 0,31 \\
\hline & Marinobacter spp. & 6,40 & 6,32 & 16,76 & 3,14 & 1,97 \\
\hline & Marinobaterium spp. & 0,78 & 1,48 & 1,09 & 1,00 & 1,09 \\
\hline & Marinomonas spp. & 0,48 & 0,94 & 0,47 & 0,96 & 0,60 \\
\hline & Methylophaga spp. & 0,19 & 0,19 & 0,22 & 0,14 & 0,30 \\
\hline & Microbulbifer spp. & 0,69 & 0,82 & 0,60 & 1,10 & 0,52 \\
\hline & Moraxella spp. & 0,42 & 0,17 & 0,06 & 0,23 & 0,37 \\
\hline & Oceanimonas spp. & 0,44 & 0,16 & 0,43 & 1,15 & 0,10 \\
\hline & Oceanospirillum spp. & 0,12 & 0,29 & 0,16 & 0,23 & 0,18 \\
\hline & Oleiphilus spp. & 0,05 & 0,03 & 0,15 & 0,04 & 0,08 \\
\hline & Oleispira spp. & 0,05 & 0,14 & 0,13 & 0,11 & 0,05 \\
\hline & Pantoea spp. & 0,30 & 0,52 & 0,36 & 1,33 & 0,63 \\
\hline & Photobacterium spp. & 0,41 & 0,86 & 0,52 & 2,15 & 0,83 \\
\hline & Pseudoalteromonas spp. & 1,27 & 0,37 & 1,92 & 0,85 & 1,63 \\
\hline & Pseudomonas spp. & 12,93 & 14,08 & 12,07 & 21,14 & 45,57 \\
\hline & Psychromonas spp. & 0,21 & 0,22 & 0,26 & 1,12 & 0,34 \\
\hline & Psychrobacter spp. & 0,64 & 0,21 & 6,81 & 0,51 & 1,26 \\
\hline & Rheinheimeria spp. & 0,42 & 0,13 & 0,10 & 0,27 & 0,45 \\
\hline & Salmonella spp. & 0,44 & 0,38 & 0,19 & 1,68 & 1,14 \\
\hline & Shewanella spp. & 2,19 & 2,78 & 2,59 & 9,12 & 3,38 \\
\hline & Thalassolituus spp. & 0,24 & 0,80 & 0,04 & 0,04 & 0,02 \\
\hline & Thalassomonas spp. & 0,24 & 0,19 & 0,10 & 0,40 & 0,29 \\
\hline & Thiomicrospira spp. & 0,26 & 0,40 & 0,63 & 0,66 & 0,40 \\
\hline & Thiorthodococcus spp. & 0,17 & 0,13 & 0,11 & 0,11 & 0,23 \\
\hline & Vibrio spp. & 0,96 & 2,38 & 1,14 & 5,08 & 3,85 \\
\hline & Xenorrabdus spp. & 0,18 & 0,60 & 0,32 & 0,33 & 0,49 \\
\hline & Methylococcaceae & 2,00 & 0,54 & 1,05 & 0,63 & 1,16 \\
\hline & Xanthomonadaceae & 2,43 & 0,20 & 0,15 & 1,63 & 0,25 \\
\hline Deltaproteobacteria & $\begin{array}{l}\text { Deltaproteobacteria } \\
\end{array}$ & 0,33 & 0,68 & 0,29 & 0,43 & 0,84 \\
\hline \multirow{2}{*}{ Flavobacteria } & Chryseobacterium spp. & 0,00 & 0,53 & 0,01 & 0,03 & 0,00 \\
\hline & Flavobacterium spp. & 0,00 & 0,37 & 0,79 & 0,10 & 0,04 \\
\hline Actinobacteria & Actinobacteria & 0,05 & 1,81 & 2,56 & 1,51 & 0,34 \\
\hline Firmicutes & Firmicutes & 1,86 & 4,86 & 7,42 & 1,04 & 0,89 \\
\hline \multirow[t]{2}{*}{ Spirochaeta } & Leptospira spp. & 0,00 & 0,03 & 0,03 & 0,06 & 0,17 \\
\hline & Others & 28,02 & 35,68 & 23,87 & 24,43 & 17,90 \\
\hline
\end{tabular}


Supplementary Table 4a: Annotation of the metagenomic data from the Aqaba UA sample from day 21, displayed in Figure 3 of the main text. The subset consists of 30 genes involved in uric acid metabolism from a total of 26,866 ORFs identified in the metagenome. Gene sequences with similarity to Halomonas spp. are printed in bold.

\begin{tabular}{|c|c|c|c|c|c|}
\hline Gene Name & Definition & Ec number & ID Best Hit & Organism & Comments \\
\hline gene_ULIXESI_12295 & $\begin{array}{l}\text { Ureidoglycolate hydrolase (EC:3.5.3.19) } \\
\end{array}$ & 3.5 .3 .19 & WP_009825456.1 & Alphaproteobacteria & Likely similar to Rhodobacteraceae \\
\hline gene_ULIXES1_19221 & Ureidoglycolate hydrolase (EC:3.5.3.19) & 3.5.3.19 & WP_004579155.1 & Gammaproteobacteria & Likely similar to Pseudomonadaceae or Alteromonadaceae - some similarity to Halomonas \\
\hline gene_ULIXES1_21068 & Ureidoglycolate hydrolase (EC:3.5.3.19) & 3.5.3.19 & WP_016855753.1 & Gammaproteobacteria & Likely similar to Halomonadaceae (Halomonas genus) \\
\hline gene_ULIXES1_21700 & Ureidogycolate hydrolase (EC.3.5.3.19) & 3.5.3.19 & NP_746404.1 & Gammaproteobacteria & Likely similar to Pseudomonadaceae (Pseudomonas genus) \\
\hline gene_ULIXES1 2453 & 5-Hydroxyisourate hydrolase & 3.5.2.17 & WP 005850231.1 & Alphaproteobacteria & Likely similar to Rhodobacteraceae \\
\hline gene_ULIXES1_2499 & 5-Hydroxyisourate hydrolase & 3.5.2.17 & WP_009828007.1 & Aphaproteobacteria & Likely similar to Rhodobacteraceae \\
\hline gene_ULIXES1_12911 & 5-Hydroxyisourate hydrolase & 3.5.2.17 & WP 022962137.1 & Gammaproteobacteria & Likely similar to Pseudomonadaceae or Alteromonadaceae \\
\hline gene_ULIXESI_14824 & 5-Hydroxyisourate hydrolase & 3.5.2.17 & WP_003298230.1 & Gammaproteobacteria & Likely similar to Pseudomonadaceae (Pseudomonas genus) \\
\hline gene_ULIXES1_21697 & 5-Hydroxyisourate hydrolase & 3.5 .2 .17 & $\mathrm{NP}_{-} 746401.1$ & Gammaproteobacteria & Likely similar to Pseudomonadaceae (Pseudomonas genus) \\
\hline gene_ULIXES1_23813 & 5-Hydroxyisourate hydrolase & 3.5.2.17 & YP_004715528.1 & Gammaproteobacteria & Likely similar to Pseudomonadaceae (Pseudomonas genus) \\
\hline gene_ULIXES1_7891 & 5-Hydroxyisourate hydrolase & & YP_001173592.1 & Gammaproteobacteria & Likely similar to Pseudomonadaceae (Pseudomonas genus) \\
\hline gene_ULIXES1_13633 & Allantoicase (EC 3.5.3.4) & 3.5.3.4 & WP_003298235.1 & Gammaproteobacteria & Likely similar to Pseudomonadaceae (Pseudomonas genus) \\
\hline gene_ULIXES1_19220 & Allantoicase (EC 3.5.3.4) & 3.5.3.4 & WP_004579156.1 & Gammaproteobacteria & Likely similar to Pseudomonadaceae or Alteromonadaceae - some similarity to Halomonas \\
\hline gene_ULIXES1_4670 & Allantoate amidohydrolase & 3.5 .1 .6 & WP_009825998.1 & Alphaproteobacteria & Likely similar to Rhodobacteraceae \\
\hline gene_ULIXESI_14474 & Allantoate amidohydrolase & 3.5.1.6 & WP 019966333.1 & Aphaproteobacteria & Likely simlar to Rhodobacteraceae or Rhizobiaceae \\
\hline gene_ULIXESI 21968 & Allantoate amidohydrolase & 3.5 .1 .6 & WP 019436796.1 & Gammaproteobacteria & Likely similar to Pseudomonadaceae (Pseudomonas genus) \\
\hline gene_ULIXESI_12294 & Allantoin catabolism protein & 3.5.3.- & WP_005850227.1 & Alphaproteobacteria & $\begin{array}{l}\text { Likely similar to Rhodobacteraceae } \\
\text {. }\end{array}$ \\
\hline gene_ULIXES1_21067 & Allantoin catabolism protein & 3.5.3.- & YP_006557201.1 & Gammaproteobacteria & Likely similar to Pseudomonadaceae or Alteromonadaceae - some similarity to Halomonas \\
\hline gene_ULIXES1_25528 & Allantoin catabolism protein & 3.5.3.- & NP_745667.1 & Gammaproteobacteria & Likely similar to Pseudomonadaceae or Alteromonadaceae - some similarity to Halomonas \\
\hline gene_ULIXES1_2451 & Urate catabolism protein/Allantoinase & & WP_005850229.1 & Alphaproteobacteria & Likely similar to Rhodobacteraceae \\
\hline gene_ULIXES1_2498 & Urate catabolism protein/Alantoinase & & WP 008886954.1 & Alphaproteobacteria & Likely similar to Rhodobacteraceae \\
\hline gene_ULIXESI_14709 & Urate catabolism protein/Alantonase & & WP_005861136.1 & Aphaproteobacteria & Ambiguous \\
\hline gene_ULIXESI_14823 & Urate catabolism protein/Allantoinase & & WP_017246039.1 & Gammaproteobacteria & $\begin{array}{l}\text { Likely similar to Pseudomonadaceae (Pseudomonas genus) }\end{array}$ \\
\hline gene_ULIXES1_19219 & Urate catabolism protein/Allantoinase & & WP_004579157.1 & Gammaproteobacteria & Likely similar to Pseudomonadaceae or Alteromonadaceae - \\
\hline gene_ULIXES1_21066 & Urate catabolism protein/Allantoinase & & WP_022963453.1 & Gammaproteobacteria & Likely similar to Pseudomonadaceae or Alteromonadaceae - some similarity to Halomonas \\
\hline gene_ULIXESI_21698 & Urate catabolism protein/Allantoinase & & WP 021783015.1 & Gammaproteobacteria & 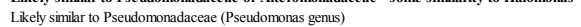 \\
\hline gene_ULIXES1_24466 & Urate catabolism protein/Allantoinase & & WP_017246039.1 & Gammaproteobacteria & Likely similar to Pseudomonadaceae (Pseudomonas genus) \\
\hline gene_ULIXES1_12912 & OHCU decarboxylase/allantoate amidohydrolase & & WP 022962138.1 & Gammaproteobacteria & Likely similar to Pseudomonadaceae or Alteromonadaceae \\
\hline gene_ULIXES1 13631 & OHCU decarboxylase/allantoate amidohydrolase & & YP 001173574.1 & Gammaproteobacteria & Likely similar to Pseudomonadaceae (Pseudomonas genus) \\
\hline gene ULIXES1 21699 & OHCU decarboxylase/allantoate amidohydrolase & & WP 021783014.1 & Gammaproteobacteria & Likely similar to Pseudomonadaceae (Pseudomonas genus) \\
\hline
\end{tabular}

Supplementary Table 4b: Annotation of the metagenomic data from the Ancona UA sample from day 21, displayed in Figure 3 of the main text. The subset consists of 15 genes involved in uric acid metabolism from a total of 27,893 ORFs identified in the metagenome. Gene sequences with similarity to Halomonas spp. are printed in bold.

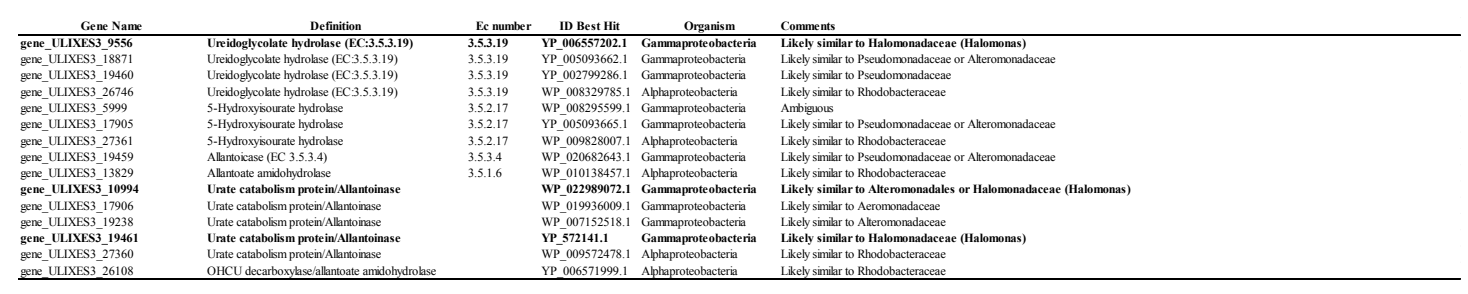

Supplementary Table 4c: Annotation of the metagenomic data from the Ancona NP sample from day 21, displayed in Figure 3 of the main text. The subset consists of 18 genes involved in uric acid metabolism from a total of 32,180 ORFs identified in the metagenome. Gene sequences with similarity to Halomonas spp. are printed in bold.

\begin{tabular}{|c|c|c|c|c|c|}
\hline Gene Name & $\begin{array}{r}\text { Definition } \\
\end{array}$ & Ec number & ID Best Hit & Organism & Comments \\
\hline gene_ULIXES-2_7300 & Ureidoglycolate hydrolase (EC:3.5.3.19) & 3.5 .3 .19 & WP_008329785.1 & Alphaproteobacteria & Likely similar to Rhodobacteraceae \\
\hline gene_ULIXES-2__10738 & Ureidoglycolate hydrolase (EC:3.5.3.19) & 3.5 .3 .19 & WP_017641447.1 & Gammaproteobacteria & Likely similar to Pseudomonadaceae or Alteromonadaceae \\
\hline gene_ULIXES-2_27693 & Ureidoglycolate hydrolase (EC 3.5.3.19) & 3.5.3.19 & WP_009572476.1 & Alphaproteobacteria & Lkely similar to Rhodobacteraceae \\
\hline gene_ULIXES-2_30028 & Ureidoglycolate hydrolase (EC 3.5.3.19) & 3.5 .3 .19 & YP_007683906.1 & Gammaproteobacteria & Likely similar to Oceanospririlales (Thalassolituus) \\
\hline gene_ULIXES-2__7302 & 5-Hydroxyisourate hydrolase & 3.5.2.17 & WP_009828007.1 & Aphaproteobacteria & Likely similar to Rhodobacteraceae \\
\hline gene_ULIXES-2_13190 & 5-Hydroxyisourate hydrolase & 3.5.2.17 & WP_008295599.1 & Gammaproteobacteria & Likely similar to Pseudomonadaceae or Alteromonadaceae \\
\hline gene_ULIXES-2_30029 & 5-Hydroxyisourate hydrolase & 3.5.2.17 & YP_007683907.1 & Gammaproteobacteria & Likely similar to Oceanospirillales (Thalassolituus) \\
\hline gene_ULIXES-2_31605 & 5-Hydroxyisourate hydrolase & 3.5 .2 .17 & WP_009572479.1 & Alphaproteobacteria & Likely similar to Rhodobacteraceae \\
\hline gene_ULIXES-2_10737 & Allantoicase (EC 3.5.3.4) & 3.5.3.4 & WP_020682643.1 & Gammaproteobacteria & Likely similar to Pseudomonadaceae or Atteromonadaceae \\
\hline gene_ULIXES-2_30031 & Allantoicase (EC 3.5.3.4) & 3.5.3.4 & YP_007683909.1 & Gammaproteobacteria & Likely similar to Oceanospirillales (Thalassolituus) \\
\hline gene_ULIXES-2_27694 & Allantoin catabolism protein & 3.5.3.- & WP_009572477.1 & Alphaproteobacteria & Likely similar to Rhodobacteraceae \\
\hline gene_ULIXES-2_7301 & Allantoinase & 3.5.2.5 & WP_008207416.1 & Alphaproteobacteria & Likely similar to Rhodobacteraceae \\
\hline gene_ULIXES-2_27695 & Allantoinase/bifinctional allantoicase $/ \mathrm{OHCU}$ decarboxylase & & WP 009572478.1 & Alphaproteobacteria & Likely similar to Rhodobacteraceae \\
\hline gene_ULIXES-2_10739 & Urate catabolism protein/Allantoinase & 3.5.2.5 & YP_572141.1 & Gammaproteobacteria & Likely similar to Halomonadaceae \\
\hline gene_ULIXES-2_10740 & Urate catabolism protein/Allantoinase & 3.5 .2 .5 & WP_017891710.1 & Proteobacteria & Ambiguous \\
\hline gene_ULIXES-2_23917 & Urate catabolism protein/Allantoinase & 3.5 .2 .5 & WP_017891710.1 & Gammaproteobacteria & Ambiguous \\
\hline gene_ULIXES-2_30025 & Urate catabolism protein/Allantoinase & & YP_-007683903.1 & Gammaproteobacteria & Likely similar to Oceanospirillales (Thalassolituus) \\
\hline gene_ULIXES-2_13189 & OHCU decarboxylase/allantoate amidohydrohse & & WP 020560622.1 & Gammaproteobacteria & Ambiguous \\
\hline gene_ULIXES-2_30030 & OHCU decarboxylase/allantoate amidohydrolase & & YP_007683908.1 & Gammaproteobacteria & Likely similar to Oceanospirillales (Thalassolituss) \\
\hline
\end{tabular}

Supplementary Table 4d: Annotation of the metagenomic data from the Bizerte NP sample from day 21, displayed in Figure 3 of the main text. The subset consists of 6 genes involved in uric acid metabolism from a total of 28,698 ORFs identified in the metagenome. Gene sequences with similarity to Halomonas spp. are printed in bold.

\begin{tabular}{|c|c|c|c|c|c|}
\hline Gene Name & Definition & Ec number & ID Best Hit & Organism & Comments \\
\hline gene_ULIXES4_4504 & Ureidoglycolate hydrolase (EC:3.5.3.19) & 3.5 .3 .19 & $\begin{array}{l}\text { YP_007683906.1 } \\
\end{array}$ & Gammaproteobacteria & Likely similar to Oceanospirillales (Thalassolituus genus) \\
\hline gene_ULIXES4_-4506 & 5-Hydroxyisourate hydrolase & 3.5.2.17 & YP_007683907.1 & Gammaproteobacteria & Likely similar to Oceanospirillales (Thalassolituus genus) \\
\hline gene_ULIXES4_-10647 & 5-Hydroxyisourate hydrolase & 3.5.2.17 & WP_008225166.1 & Alphaproteobacteria & Likely similar to Rhodobacteraceae \\
\hline gene_ULIXES4_4508 & Allantoicase (EC 3.5.3.4) & 3.5.3.4 & YP_007683909.1 & Gammaproteobacteria & Likely similar to Oceanospirillales (Thalassolituus genus) \\
\hline gene_ULIXES4_4501 & Urate catabolism protein/Allantoinase & & YP_007683903.1 & Gammaproteobacteria & Likely similar to Oceanospirillales (Thalassolituus genus) \\
\hline gene_ULIXES4-4507 & OHCU decarboxylase/allantoate amidohydrolase & & YP_007683908.1 & Gammaproteobacteria & Likely similar to Oceanospirillales (Thalassolituus genus) \\
\hline
\end{tabular}


Supplementary Table 4e: Annotation of the metagenomic data from the El Max UA sample from day 21, displayed in Figure 3 of the main text. The subset consists of 29 genes involved in uric acid metabolism from a total of 61,277 ORFs identified in the metagenome. Gene sequences with similarity to Halomonas spp. are printed in bold.

\begin{tabular}{|c|c|c|c|c|c|}
\hline Gene Name & Definition & Ec number & ID Best Hit & Organism & Comments \\
\hline gene_ULIXES5_16814 & Ureidoglycolate hydrolase (EC:3.5.3.19) & 3.5 .3 .19 & WP_007482915.1 & Gammaproteobacteria & Likely similar to Pseudomonadales (Acinetobacter genus) \\
\hline gene_ULIXES5_22395 & Ureidoglycolate hydrolase (EC:3.5.3.19) & 3.5.3.19 & NP_746404.1 & Gammaproteobacteria & Likely similar to Pseudomonadales (Pseudomonas genus) \\
\hline gene_ULIXES5_29775 & Ureidoglycolate hydrolase (EC:3.5.3.19) & 3.5.3.19 & YP_001534537.1 & Alphaproteobacteria & Likely similar to Rhodobacteraceae \\
\hline gene_ULIXES5_30682 & Ureidoglycolate hydrolase (EC:3.5.3.19) & 3.5.3.19 & WP_016855753.1 & Gammaproteobacteria & Likely similar to Halomonadaceae (Halomonas) \\
\hline gene_ULIXES5_47410 & Ureidoglycolate hydrolase (EC:3.5.3.19) & 3.5.3.19 & YP_005093662.1 & Gammaproteobacteria & Likely similar to Aeromonadaceae - some similarity to Halomonas \\
\hline gene_ULIXES5_-77641 & Ureidoglycolate hydrolase (EC:3.5.3.19) & 3.5.3.19 & WP_-003299751.1 & Gammaproteobacteria & Likely similar to Pseudomonadales (Pseudomonas genus) \\
\hline gene_ULIXES5_17480 & 5-Hydroxyisourate hydrolase & 3.5.2.17 & WP_007481680.1 & Gammaproteobacteria & Likely similar to Pseudomonadales (Acinetobacter genus) \\
\hline gene_ULIXES5_29777 & 5-Hydroxyisourate hydrolase & 3.5.2.17 & WP_007803838.1 & Alphaproteobacteria & Likely similar to Rhodobacteraceae \\
\hline gene_ULIXES5_29869 & 5-Hydroxyisourate hydrolase & 3.5.2.17 & YP_004715528.1 & Gammaproteobacteria & Likely similar to Pseudomonadales (Pseudomonas genus) - some similarity to Halomonas \\
\hline gene_ULIXES5_41564 & 5-Hydroxyisourate hydrolase & 3.5.2.17 & YP_004715528.1 & Gammaproteobacteria & Likely similar to Pseudomonadales (Pseudomonas genus) - some similarity to Halomonas \\
\hline gene_ULIXES5_-̄ 45441 & 5-Hydroxyisourate hydrolase & 3.5.2.17 & WP_-008566757.1 & Gammaproteobacteria & Likely similar to Pseudomonadales (Pseudomonas genus) \\
\hline gene_ULIXES5_47413 & 5-Hydroxyisourate hydrolase & 3.5.2.17 & YP_005093665.1 & Gammaproteobacteria & Likely similar to Aeromonadaceae - some similarity to Halomonas \\
\hline gene_ULIXES5_49758 & 5-Hydroxyisourate hydrolase & 3.5.2.17 & WP_004880295.1 & Gammaproteobacteria & Likely similar to Pseudomonadales (Acinetobacter genus) \\
\hline gene_ULIXESS_56324 & 5-Hydroxyisourate hydrolase & 3.5.2.17 & YP_005091502.1 & Gammaproteobacteria & Likely similar to Aeromonadaceae - some similarity to Halomonas \\
\hline gene_ULIXES5_16815 & Allantoicase (EC 3.5.3.4) & 3.5.3.4 & WP_007482917.1 & Gammaproteobacteria & Likely similar to Pseudomonadales (Acinetobacter genus) \\
\hline gene_ULIXES5_28263 & Allantoin catabolism protein & 3.5.3.- & YP_005090755.1 & Gammaprote obacteria & Likely similar to Aeromonadaceae - some similarity to Halomonas \\
\hline gene_ULIXES5_29772 & Allantoin catabolism protein & 3.5.3.- & WP__009816826.1 & Alphaproteobacteria & Likely similar to Rhodobacteraceae \\
\hline gene_ULIXES5__30681 & Allantoin catabolism protein & 3.5.3.- & YP_006557201.1 & Gammaproteobacteria & Likely similar to Pseudomonadaceae or Alteromonadaceae - some similarity to Halomonas \\
\hline gene_ULIXES5_29776 & Allantoinase/bifinctional allantoicase $/ \mathrm{OHCU}$ decarboxylase & & YP_001534538.1 & Alphaproteobacteria & Likely similar to Rhodobacteraceae \\
\hline gene_ULIXES5_16808 & Urate catabolism protein/Allantoinase & & WP_-004880270.1 & Gammaproteobacteria & Likely similar to Pseudomonadales (Acinetobacter genus) \\
\hline gene_ULIXESS__30680 & Urate catabolism protein/Allantoinase & & WP_019019214.1 & Gammaproteobacteria & Likely similar to Halomonadaceae (Halomonas) \\
\hline gene_ULIXES5_45440 & Urate catabolism protein/Allantoinase & & WP_017246039.1 & Gammaproteobacteria & Likely similar to Pseudomonadales (Pseudomonas genus) \\
\hline gene_ULIXES5_45741 & Urate catabolism protein/Allantoinase & & WP_004579157.1 & Proteobacteria & Ambiguous \\
\hline gene_ULIXES5 547412 & Urate catabolism protein/Allantoinase & & YP_005093664.1 & Gammaproteobacteria & Likely similar to Aeromonadaceae \\
\hline gene_ULIXES5__48158 & Urate catabolism protein/Allantoinase & & WP__004579157.1 & Gammaproteobacteria & Likely similar to Alteromonadales or Halomonadace ae (I \\
\hline gene_ULIXES5_22394 & $\mathrm{OHCU}$ decarboxylase/allantoate amidohydrolsse & & WP_-021783014.1 & Gammaproteobacteria & Likely similar to Pseudomonadales (Pseudomonas genus) \\
\hline gene_ULIXES5_47411 & OHCU decarboxylase/allantoate amidohydrolase & & YP_005093663.1 & Gammaproteobacteria & Likely similar to Aeromonadaceae \\
\hline & & & & & \\
\hline gene ULIXES5 53891 & OHCU decarboxylase/allantoate amidohydrolase & & WP 017246038.1 & Gammaproteobacteria & Likely similar to Pseudomonadales (Pseudomonas genus) \\
\hline
\end{tabular}


2 Supplementary Table 5: Closest relatives of 16S rRNA gene sequences of uric acid-degrading 3 isolates. The first letter of the isolate code designates the sediment sampling location (A - Ancona; Q 4 Aqaba; X - El Max; B - Bizerte), and the second letter indicates the nitrogen source ( $\mathrm{N}$ - Ammonium; 5 U- Uric Acid). The first number in the isolate code represents the sampling date, and the last number 6 indicates the individual isolate.

\begin{tabular}{|c|c|c|c|c|}
\hline Sequence & Closest relative & Accession nr. & Phylogeny & Seq. identity $(\%)$ \\
\hline AN12-1 & Halomonas venusta & NR_042069 & Bacteria; Proteobacteria; Gammaproteobacteria; Oceanospirillales; Halomonadaceae & $96 \%$ \\
\hline AN12-2 & Halomonas alkaliphila & NR_042256 & Bacteria; Proteobacteria; Gammaproteobacteria; Oceanospirillales; Halomonadaceae & $99 \%$ \\
\hline AN12-3 & Halomonas venusta & AY669165 & Bacteria; Proteobacteria; Gammaproteobacteria; Oceanospirillales; Halomonadaceae & $99 \%$ \\
\hline AN12-4 & Halomonas alkaliphila & NR_042256 & Bacteria; Proteobacteria; Gammaproteobacteria; Oceanospirillales; Halomonadaceae & $100 \%$ \\
\hline AU6-1 & Halomonas venusta & JF710982 & Bacteria; Proteobacteria; Gammaproteobacteria; Oceanospirillales; Halomonadaceae & $99 \%$ \\
\hline AU6-2 & Halomonas alkaliphila & NR_042256 & Bacteria; Proteobacteria; Gammaproteobacteria; Oceanospirillales; Halomonadaceae & $100 \%$ \\
\hline AU6-3 & Halomonas venusta & JF710982 & Bacteria; Proteobacteria; Gammaproteobacteria; Oceanospirillales; Halomonadaceae & $99 \%$ \\
\hline AU6-4 & Halomonas alkaliphila & JQ680444 & Bacteria; Proteobacteria; Gammaproteobacteria; Oceanospirillales; Halomonadaceae & $99 \%$ \\
\hline AU12-1 & Halomonas alkaliphila & NR_042256 & Bacteria; Proteobacteria; Gammaproteobacteria; Oceanospirillales; Halomonadaceae & $99 \%$ \\
\hline AU12-2 & Halomonas venusta & AY669165 & Bacteria; Proteobacteria; Gammaproteobacteria; Oceanospirillales; Halomonadaceae & $99 \%$ \\
\hline AU12-3 & Halomonas venusta & AY669165 & Bacteria; Proteobacteria; Gammaproteobacteria; Oceanospirillales; Halomonadaceae & $99 \%$ \\
\hline AU12-4 & Halomonas alkaliphila & NR_042256 & Bacteria; Proteobacteria; Gammaproteobacteria; Oceanospirillales; Halomonadaceae & $100 \%$ \\
\hline BN12-1 & Halomonas salifodinae & NR_044263 & Bacteria; Proteobacteria; Gammaproteobacteria; Oceanospirillales; Halomonadaceae & $99 \%$ \\
\hline BN12-2 & Marinobacter adhaerens & NR_074765 & Bacteria; Proteobacteria; Gammaproteobacteria; Alteromonadales; Alteromonadaceae & $87 \%$ \\
\hline BN12-3 & Halomonas denitrificans & EU541350 & Bacteria; Proteobacteria; Gammaproteobacteria; Oceanospirillales; Halomonadaceae & $98 \%$ \\
\hline BN12-4 & Marinobacter lipolyticus & NR_025671 & Bacteria; Proteobacteria; Gammaproteobacteria; Alteromonadales; Alteromonadaceae & $99 \%$ \\
\hline BU6-1 & Halomonas salifodinae & NR_044263 & Bacteria; Proteobacteria; Gammaproteobacteria; Oceanospirillales; Halomonadaceae & $99 \%$ \\
\hline BU6-2 & Halomonas venusta & NR_042069 & Bacteria; Proteobacteria; Gammaproteobacteria; Oceanospirillales; Halomonadaceae & $99 \%$ \\
\hline BU6-3 & Halomonas neptunia & KC534295 & Bacteria; Proteobacteria; Gammaproteobacteria; Oceanospirillales; Halomonadaceae & $99 \%$ \\
\hline BU6-4 & Halomonas salifodinae & NR_044263 & Bacteria; Proteobacteria; Gammaproteobacteria; Oceanospirillales; Halomonadaceae & $98 \%$ \\
\hline BU12-1 & Halomonas salifodinae & NR_044263 & Bacteria; Proteobacteria; Gammaproteobacteria; Oceanospirillales; Halomonadaceae & $99 \%$ \\
\hline BU12-2 & Halomonas venusta & JF710984 & Bacteria; Proteobacteria; Gammaproteobacteria; Oceanospirillales; Halomonadaceae & $99 \%$ \\
\hline BU12-3 & Halomonas salifodinae & NR_044263 & Bacteria; Proteobacteria; Gammaproteobacteria; Oceanospirillales; Halomonadaceae & $99 \%$ \\
\hline BU12-4 & Halomonas salifodinae & NR_044263 & Bacteria; Proteobacteria; Gammaproteobacteria; Oceanospirillales; Halomonadaceae & $99 \%$ \\
\hline QN12-1 & Halomonas venusta & AY669165 & Bacteria; Proteobacteria; Gammaproteobacteria; Oceanospirillales; Halomonadaceae & $99 \%$ \\
\hline QN12-2 & Halomonas venusta & AY669165 & Bacteria; Proteobacteria; Gammaproteobacteria; Oceanospirillales; Halomonadaceae & $99 \%$ \\
\hline QN12-3 & Halomonas venusta & NR_042069 & Bacteria; Proteobacteria; Gammaproteobacteria; Oceanospirillales; Halomonadaceae & $99 \%$ \\
\hline QN12-4 & Halomonas venusta & AY 669165 & Bacteria; Proteobacteria; Gammaproteobacteria; Oceanospirillales; Halomonadaceae & $99 \%$ \\
\hline QU6-1 & Halomonas venusta & AY669165 & Bacteria; Proteobacteria; Gammaproteobacteria; Oceanospirillales; Halomonadaceae & $99 \%$ \\
\hline QU6-2 & Halomonas venusta & AY669165 & Bacteria; Proteobacteria; Gammaproteobacteria; Oceanospirillales; Halomonadaceae & $99 \%$ \\
\hline QU6-3 & Halomonas venusta & AY669165 & Bacteria; Proteobacteria; Gammaproteobacteria; Oceanospirillales; Halomonadaceae & $99 \%$ \\
\hline QU6-4 & Halomonas venusta & AY669165 & Bacteria; Proteobacteria; Gammaproteobacteria; Oceanospirillales; Halomonadaceae & $99 \%$ \\
\hline QU12-1 & Halomonas nitroreducens & NR_044317 & Bacteria; Proteobacteria; Gammaproteobacteria; Oceanospirillales; Halomonadaceae & $98 \%$ \\
\hline QU12-2 & Halomonas salifodinae & JN645859 & Bacteria; Proteobacteria; Gammaproteobacteria; Oceanospirillales; Halomonadaceae & $99 \%$ \\
\hline QU12-3 & Halomonas venusta & AY 669165 & Bacteria; Proteobacteria; Gammaproteobacteria; Oceanospirillales; Halomonadaceae & $99 \%$ \\
\hline QU12-4 & Halomonas venusta & AY669165 & Bacteria; Proteobacteria; Gammaproteobacteria; Oceanospirillales; Halomonadaceae & $99 \%$ \\
\hline XN12-1 & Halomonas nitroreducens & NR_044317 & Bacteria; Proteobacteria; Gammaproteobacteria; Oceanospirillales; Halomonadaceae & $98 \%$ \\
\hline $\mathrm{XN} 12-2$ & Halomonas ventosae & FJ444984 & Bacteria; Proteobacteria; Gammaproteobacteria; Oceanospirillales; Halomonadaceae & $98 \%$ \\
\hline $\mathrm{XN} 12-3$ & Halomonas ventosae & FJ444984 & Bacteria; Proteobacteria; Gammaproteobacteria; Oceanospirillales; Halomonadaceae & $98 \%$ \\
\hline $\mathrm{XN} 12-4$ & Halomonas ventosae & FJ444984 & Bacteria; Proteobacteria; Gammaproteobacteria; Oceanospirillales; Halomonadaceae & $98 \%$ \\
\hline XU6-1 & Pelomonas aquatica & NR_042614 & Bacteria; Proteobacteria; Betaproteobacteria; Burkholderiales; Comamonadaceae & $99 \%$ \\
\hline XU6-2 & Halomonas neptunia & KC354707 & Bacteria; Proteobacteria; Gammaproteobacteria; Oceanospirillales; Halomonadaceae & $99 \%$ \\
\hline XU6-4 & Halomonas ventosae & NR_042812 & Bacteria; Proteobacteria; Gammaproteobacteria; Oceanospirillales; Halomonadaceae & $99 \%$ \\
\hline XU12-1 & Halomonas ventosae & FJ444984 & Bacteria; Proteobacteria; Gammaproteobacteria; Oceanospirillales; Halomonadaceae & $98 \%$ \\
\hline XU12-2 & Halomonas denitrificans & EU541350 & Bacteria; Proteobacteria; Gammaproteobacteria; Oceanospirillales; Halomonadaceae & $97 \%$ \\
\hline XU12-3 & Halomonas ventosae & FJ444984 & Bacteria; Proteobacteria; Gammaproteobacteria; Oceanospirillales; Halomonadaceae & $97 \%$ \\
\hline XU12-4 & Halomonas ventosae & FJ444984 & Bacteria; Proteobacteria; Gammaproteobacteria; Oceanospirillales; Halomonadaceae & $98 \%$ \\
\hline
\end{tabular}

